\title{
VANISHING IDEALS OVER GRAPHS AND EVEN CYCLES
}

\author{
JORGE NEVES, MARIA VAZ PINTO, AND RAFAEL H. VILLARREAL
}

\begin{abstract}
Let $X$ be an algebraic toric set in a projective space over a finite field. We study the vanishing ideal, $I(X)$, of $X$ and show some useful degree bounds for a minimal set of generators of $I(X)$. We give an explicit combinatorial description of a set of generators of $I(X)$, when $X$ is the algebraic toric set associated to an even cycle or to a connected bipartite graph with pairwise vertex disjoint even cycles. In this case, a formula for the regularity of $I(X)$ is given. We show an upper bound for this invariant, when $X$ is associated to a (not necessarily connected) bipartite graph. The upper bound is sharp if the graph is connected. We are able to show a formula for the length of the parameterized linear code associated with any graph, in terms of the number of bipartite and non-bipartite components.
\end{abstract}

\section{INTRODUCTION}

Let $\mathbb{P}^{s-1}$ be a projective space over a finite field $\mathbb{F}_{q}$. An evaluation code, also known as a generalized Reed-Muller code, is a linear code obtained by evaluating the linear space of homogeneous $d$-forms on a set of points $X \subset \mathbb{P}^{s-1}$ (see Definition 2.1). A linear code obtained in this way, denoted by $C_{X}(d)$, has length $|X|$. Evaluation codes have been the object of much attention in recent years. To describe their basic parameters (length, dimension and minimum distance), many authors have been using tools coming from Algebraic Geometry and Commutative Algebra, see [2, 3, 7, 11, 17, 19, 22, Let $\mathbb{T}^{s-1}$ be a projective torus in $\mathbb{P}^{s-1}$. A parameterized linear code is a special type of generalized Reed-Muller code obtained when $X \subset \mathbb{T}^{s-1} \subset \mathbb{P}^{s-1}$ is parameterized by a set of monomials (see Definition 2.5), in this case $X$ is called an algebraic toric set because it generalizes the notion of a projective torus. Parameterized linear codes were introduced and studied in [15. The extra structure on $X$ yields alternative methods to compute the basic parameters of $C_{X}(d)$.

In this article we focus on linear codes parameterized by the edges of a graph $\mathcal{G}$ (see Definition 2.6). For the study of algebraic toric sets parameterized by the edges of a clutter, which is a natural generalization of the concept of graph, we refer the reader to [17, 18. Not much is known about the parameterized linear codes associated to a general graph. The first results in this direction appear in [10], where the length, dimension and minimum distance of the codes associated to complete bipartite graphs are computed. In [15], one can find a formula for the length of the code associated to a connected graph (see this formula in Proposition 2.7) and also a bound for the minimum distance of the code associated to a connected non-bipartite graph.

An important algebraic invariant associated to a parameterized linear code is the regularity of the ring $S / I(X)$, where $S$ is the coordinate ring of $\mathbb{P}^{s-1}$, i.e., a polynomial ring in $s$ variables, and $I(X)$ is the vanishing ideal of $X$ (see Definition 2.2). The knowledge of the regularity of $S / I(X)$ is important for applications to coding theory: for $d \geq \operatorname{reg} S / I(X)$ the code $C_{X}(d)$

2010 Mathematics Subject Classification. Primary 13P25; Secondary 14G50, 14G15, 11T71, 94B27, $94 \mathrm{~B} 05$.

The first author was partially supported by CMUC and FCT (Portugal), through European program COMPETE/FEDER. The second author is a member of the Center for Mathematical Analysis, Geometry and Dynamical Systems. The third author was partially supported by SNI. 
coincides with the underlying vector space $\mathbb{F}_{q}^{|X|}$ and has, accordingly, minimum distance equal to 1. In [24, Corollary 2.31] the authors give bounds for the regularity of $S / I(X)$, when $X$ is the algebraic toric set associated to a connected bipartite graph. In [8] a bound is given for the minimum distance of the codes associated to a graph isomorphic to a cycle of even length, as well as another bound for reg $S / I(X)$ in this case.

The contents of this paper are as follows. In Section 2, we recall the necessary background. To the best of our knowledge, there is no information available on the parameterized codes arising from disconnected graphs. If $\mathcal{G}$ is an arbitrary graph, in Section 3, Theorem 3.2, we show our first main result, an explicit formula for the length of $C_{X}(d)$ in terms of the number of bipartite and non-bipartite connected components of the graph.

An earlier result of [15] shows that the vanishing ideal $I(X)$ is minimally generated by a finite set of homogeneous binomials. In Section 4 , we study $I(X)$ for an arbitrary algebraic toric set $X$ and show some useful degree bounds for a minimal set of generators of $I(X)$ (see Theorem 4.5 and Proposition 4.6). If the graph $\mathcal{G}$ is an even cycle, another main result of this article is an explicit combinatorial description of a generating set for $I(X)$ consisting of binomials (see Theorem [5.9). This result is generalized to any connected bipartite graph whose cycles are vertex disjoint (see Theorem 5.13). We give examples of bipartite graphs not satisfying this assumption for which $I(X)$ is not generated by the set prescribed in Theorem 5.13 (see Example 5.14).

If the graph $\mathcal{G}$ is an even cycle of length $2 k$, using our description of a generating set for $I(X)$, we derive the following formula for the regularity:

$$
\operatorname{reg} S / I(X)=(q-2)(k-1)
$$

(see Theorem 6.2). Then, we give the following upper bound for the regularity of $S / I(X)$ for a general (not necessarily connected) bipartite graph with $s$ edges and $m$ cycles, with disjoint edge sets, of orders $2 k_{1}, \ldots, 2 k_{m}$ :

$$
\operatorname{reg} S / I(X) \leq(q-2)\left(s-\sum_{i=1}^{m} k_{i}-1\right)
$$

(see Theorem 6.3). In Corollary 6.5, we show that this estimate is the actual value of reg $S / I(X)$ if $\mathcal{G}$ is a connected bipartite graph with $s$ edges and with exactly $m$ even cycles, with disjoint vertex sets, of orders $2 k_{1}, \ldots, 2 k_{m}$.

The computational algebra techniques of [15] played an important role in discovering some of the results, conjectures, and examples of this paper. Using the computer algebra system Macaulay2 [12] and the results of [15], one can compute the reduced Gröbner basis, the degree and the regularity of a vanishing ideal $I(X)$ of an algebraic toric set $X$ over a finite field $\mathbb{F}_{q}$. This allows us to study and to gain insight on the algebraic invariants of a vanishing ideal that are useful in algebraic coding theory.

For all unexplained terminology and additional information, we refer to [1] (for graph theory), [5] (for the theory of binomial ideals), 4, 13, 20] (for commutative algebra and the theory of Hilbert functions), and [21, 23] (for the theory of linear codes and evaluation codes).

\section{Preliminaries}

Let $K=\mathbb{F}_{q}$ be a finite field of order $q$ and fix $s$ a positive integer. Recall that the projective space of dimension $s-1$ over $K$, denoted by $\mathbb{P}^{s-1}$, is the quotient space $\left(K^{s} \backslash\{0\}\right) / \sim$ where two vectors $\mathbf{x}_{1}, \mathbf{x}_{2}$ in $K^{s} \backslash\{0\}$ are equivalent if $\mathbf{x}_{1}=\lambda \mathbf{x}_{2}$ for some $\lambda \in K^{*}=K \backslash\{0\}$. Denote by $\mathbb{T}^{s-1}$ the subset of $\mathbb{P}^{s-1}$ given by

$$
\mathbb{T}^{s-1}=\left\{[\mathbf{x}]=\left[\left(x_{1}, \ldots, x_{s}\right)\right] \in \mathbb{P}^{s-1}: x_{1} \cdots x_{s} \neq 0\right\},
$$


where $[\mathbf{x}]$ is the equivalent class of $\mathbf{x}$. The projective torus $\mathbb{T}^{s-1}$ is an Abelian group under componentwise multiplication and is isomorphic to the standard $(s-1)$-dimensional torus, $\left(K^{*}\right)^{s-1}$, over $K$.

Consider $S=K\left[t_{1}, \ldots, t_{s}\right]=\bigoplus_{d=0}^{\infty} S_{d}$, a polynomial ring over the field $K$ with the standard grading. Given a nonempty set of points $X=\left\{\left[\mathbf{x}_{1}\right], \ldots,\left[\mathbf{x}_{m}\right]\right\} \subset \mathbb{T}^{s-1} \subset \mathbb{P}^{s-1}$ and letting $f_{0}=t_{1}$, consider, for each $d$, the map: $\mathrm{ev}_{d}: S_{d} \rightarrow K^{|X|}$ given by

$$
f \mapsto\left(\frac{f\left(\mathbf{x}_{1}\right)}{f_{0}^{d}\left(\mathbf{x}_{1}\right)}, \ldots, \frac{f\left(\mathbf{x}_{m}\right)}{f_{0}^{d}\left(\mathbf{x}_{m}\right)}\right), \quad \forall f \in S_{d} .
$$

For each $d \geq 0, \mathrm{ev}_{\mathrm{d}}$ is a linear map of $K$-vector spaces. Its image is denoted by $C_{X}(d)$.

Definition 2.1. The evaluation code of order $d$ associated to $X$ is the linear subspace of $K^{|X|}$ given by $C_{X}(d)$, for $d \geq 0$.

Notice that if $q=2$ then $\mathbb{T}^{s-1}$ is a point and, accordingly, $C_{X}(d)=K$, for all $d$. For this reason, throughout this article we assume that $q>2$.

Clearly an evaluation code is a linear code, i.e., it is a linear subspace of $K^{|X|}$. Accordingly, one defines the dimension of the code as its dimension as a vector space, i.e., as $\operatorname{dim}_{K} C_{X}(d)$, its length as the dimension of the ambient vector space, which, for evaluation codes, coincides with $|X|$ and, finally, its minimum distance, is defined as:

$$
\delta_{X}(d)=\min \left\{\|\mathbf{w}\|: 0 \neq \mathbf{w} \in C_{X}(d)\right\},
$$

where $\|\mathbf{w}\|$ is the number of nonzero coordinates of $\mathbf{w}$. The basic parameters of $C_{X}(d)$ are related by the Singleton bound for the minimum distance:

$$
\delta_{X}(d) \leq|X|-\operatorname{dim}_{K} C_{X}(d)+1 .
$$

Two of the basic parameters of $C_{X}(d)$, the dimension and the length, can be expressed using the Hilbert function of the quotient of $S$ by a particular homogeneous ideal. This ideal is the vanishing ideal of $X$, i.e., the ideal of $S$ generated by the homogeneous polynomials of $S$ that vanish on $X$. Denote it by $I(X)$. Recall that the Hilbert function of $S / I(X)$ is given by

$$
H_{X}(d):=\operatorname{dim}_{K}(S / I(X))_{d}=\operatorname{dim}_{K} S_{d} / I(X)_{d}=\operatorname{dim}_{K} C_{X}(d),
$$

see [20]. The unique polynomial $h_{X}(t)=\sum_{i=0}^{k-1} c_{i} t^{i} \in \mathbb{Q}[t]$ of degree $k-1=\operatorname{dim} S / I(X)-1$ such that $h_{X}(d)=H_{X}(d)$ for $d \gg 0$ is called the Hilbert polynomial of $S / I(X)$. The integer $c_{k-1}(k-1)$ !, denoted by $\operatorname{deg} S / I(X)$, is called the degree or multiplicity of $S / I(X)$. In our situation $h_{X}(t)$ is a nonzero constant because $S / I(X)$ has dimension 1 . Furthermore $h_{X}(d)=|X|$ for $d \geq|X|-1$, see [13, Lecture 13] and [6]. This means that $|X|$ is equal to the degree of $S / I(X)$.

A good parameterized code should have large $|X|$ together with $\operatorname{dim}_{K} C_{X}(d) /|X|$ and $\delta_{X}(d) /|X|$ as large as possible. Here, another algebraic invariant gives an indication of where to look for nontrivial evaluation codes.

Definition 2.2. The index of regularity of $S / I(X)$, denoted by reg $S / I(X)$, is the least integer $\ell \geq 0$ such that $h_{X}(d)=H_{X}(d)$ for $d \geq \ell$.

As $S / I(X)$ is a 1-dimensional Cohen-Macaulay graded algebra [6], the index of regularity of $S / I(X)$ is the Castelnuovo-Mumford regularity of $S / I(X)$ [4]. We will refer to $\operatorname{reg}(S / I(X))$ simply as the regularity of $S / I(X)$. The regularity is related to the degrees of a minimal generating set of $I(X)$. 
Definition 2.3. Let $f_{1}, \ldots, f_{r}$ be a minimal homogeneous generating set of $I(X)$. The big degree of $I(X)$ is defined as bigdeg $I(X)=\max _{i}\left\{\operatorname{deg}\left(f_{i}\right)\right\}$.

From the definition of the Castelnuovo-Mumford regularity of $S / I(X)$ [4], one has:

Proposition 2.4. bigdeg $I(X)-1 \leq \operatorname{reg}(S / I(X))$.

Since $\operatorname{dim}_{K} C_{X}(d)=H_{X}(d)$ and the Hilbert polynomial of $S / I(X)$ is a constant polynomial with constant term equal to the dimension of the ambient vector space, $K^{|X|}$, we deduce that for $d \geq \operatorname{reg} S / I(X)$ the linear code $C_{X}(d)$ coincides with $K^{|X|}$. This can also be expressed by $\delta_{X}(d)=1$ for all $d \geq \operatorname{reg} S / I(X)$. We conclude that the potentially good codes $C_{X}(d)$ can occur only if $1 \leq d<\operatorname{reg}(S / I(X))$.

For a particular class of evaluation codes, called parameterized linear codes, the ideal $I(X)$ has been studied to an extent that it is possible to use algebraic methods, based on elimination theory and Gröbner bases, to compute the dimension and the length of $C_{X}(d)$, see [15]. Let us briefly describe the notion of a parameterized linear code.

Given an $n$-tuple of integers, $\nu=\left(r_{1}, \ldots, r_{n}\right) \in \mathbb{Z}^{n}$, and a vector $\mathbf{x}=\left(x_{1}, \ldots, x_{n}\right) \in\left(K^{*}\right)^{n}$, we set $\mathbf{x}^{\nu}=x_{1}^{r_{1}} \cdots x_{n}^{r_{n}} \in K^{*}$. Let $\nu_{1}, \ldots, \nu_{s} \in \mathbb{Z}^{n}$ and let $X^{*} \subset\left(K^{*}\right)^{s}$ be the set given by:

$$
X^{*}=\left\{\left(\mathbf{x}^{\nu_{1}}, \ldots, \mathbf{x}^{\nu_{s}}\right): \mathbf{x} \in\left(K^{*}\right)^{n}\right\} .
$$

Consider the multiplicative group structure of $\left(K^{*}\right)^{s}$ and let $\pi:\left(K^{*}\right)^{s} \rightarrow \mathbb{T}^{s-1}$ be the quotient map by the diagonal subgroup $\Lambda=\left\{(\lambda, \ldots, \lambda) \in\left(K^{*}\right)^{s}: \lambda \in K^{*}\right\}$. Notice that $\mathbb{T}^{s-1}=\left(K^{*}\right)^{s} / \Lambda$ is the projective torus in $\mathbb{P}^{s-1}$.

Definition 2.5 ([16], [15]). Let $\nu_{1}, \ldots, \nu_{s} \in \mathbb{N}^{n}$. The set of points given by $X=\pi\left(X^{*}\right)$ is called an algebraic toric set parameterized by $\nu_{1}, \ldots, \nu_{s} \in \mathbb{N}^{n}$. The evaluation codes $C_{X}(d)$ obtained from an algebraic toric set $X$ are called parameterized linear codes.

It is clear that $X^{*}$ is a subgroup of $\left(K^{*}\right)^{s}$, since it is the image of the group homomorphism $\left(K^{*}\right)^{n} \rightarrow\left(K^{*}\right)^{s}$ given by $\mathbf{x} \mapsto\left(\mathbf{x}^{\nu_{1}}, \ldots, \mathbf{x}^{\nu_{s}}\right)$. Denote by $\theta:\left(K^{*}\right)^{n} \rightarrow X^{*}$ and by $\tilde{\pi}: X^{*} \rightarrow X$ the restrictions of the corresponding homomorphisms. Thus, we have the following sequence:

$$
\left(K^{*}\right)^{n} \stackrel{\theta}{\longrightarrow} X^{*} \stackrel{\widetilde{\pi}}{\longrightarrow} X \longrightarrow 1 .
$$

For a parameterized algebraic toric set $X$, the vanishing ideal $I(X)$ carries extra structure. We know that, in this situation, $I(X)$ is 1-dimensional Cohen-Macaulay lattice ideal [15]. In particular $I(X)$ is a binomial ideal, i.e., it is generated by binomials. Recall that a binomial in $S$ is a polynomial of the form $t^{a}-t^{b}$, where $a, b \in \mathbb{N}^{s}$ and where, if $a=\left(a_{1}, \ldots, a_{s}\right) \in \mathbb{N}^{s}$, we set

$$
t^{a}=t_{1}^{a_{1}} \cdots t_{s}^{a_{s}} \in S .
$$

A binomial of the form $t^{a}-t^{b}$ is usually referred to as a pure binomial [5], although here we are dropping the adjective "pure".

Let $\mathcal{G}$ be a simple graph with vertex set $V_{\mathcal{G}}=\left\{v_{1}, \ldots, v_{n}\right\}$ and edge set $E_{\mathcal{G}}=\left\{e_{1}, \ldots, e_{s}\right\}$. Throughout the remainder of this article, when dealing with a graph, we shall reserve the use of $n$ and $s$ for the number of vertices and the number of edges of the graph in question. For an edge $e_{i}=\left\{v_{j}, v_{k}\right\}$, where $v_{j}, v_{k} \in V_{\mathcal{G}}$, let $\nu_{i}=\mathbf{e}_{j}+\mathbf{e}_{k} \in \mathbb{N}^{n}$, where, for $1 \leq j \leq n$, $\mathbf{e}_{j}$ is the $j$-th element of the canonical basis of $\mathbb{Q}^{n}$.

Definition 2.6 ([10]). The algebraic toric set associated to $\mathcal{G}$ is the toric set parameterized by the $n$-tuples $\nu_{1}, \ldots, \nu_{s} \in \mathbb{N}^{n}$, obtained from the edges of $\mathcal{G}$. If $X$ is the parameterized toric set associated to $\mathcal{G}$ we call its associated linear code $C_{X}(d)$ the parameterized code associated to $\mathcal{G}$ and we refer to the vanishing ideal of $X$ as the vanishing ideal over $\mathcal{G}$. 
If $\mathbf{x}=\left(x_{1}, \ldots, x_{n}\right) \in\left(K^{*}\right)^{n}$ and $e_{i}=\left\{v_{j}, v_{k}\right\}$ is an edge of $\mathcal{G}$, we set $\mathbf{x}^{e_{i}}=\mathbf{x}^{\mathbf{e}_{j}+\mathbf{e}_{k}}=x_{j} x_{k}$, so that the structural map $\theta:\left(K^{*}\right)^{n} \rightarrow X^{*}$ is given by $\mathbf{x} \mapsto\left(\mathbf{x}^{e_{1}}, \ldots, \mathbf{x}^{e_{s}}\right)$. It is clear that if $\mathcal{G}$ contains isolated vertices, then the associated algebraic toric set $X$ coincides with the algebraic toric set associated to the subgraph of $\mathcal{G}$ obtained by removing these vertices. If $\mathcal{G}$ has a second edge through two vertices, then $X$ is isomorphic to its projection away from the coordinate point of $\mathbb{P}^{s-1}$ corresponding to that edge; which, in turn, coincides with the algebraic toric set defined by the graph obtained from $\mathcal{G}$ by removing the multiple edge. Hence, from the point of view of the algebraic toric set $X$, the existence of multiple edges in $\mathcal{G}$ is not interesting. If $\mathcal{G}$ has only one edge then is easy to see that $X=\mathbb{P}^{s-1}$ is a point, $I(X)=0$ and $C_{X}(d)=K^{*}$. Thus throughout the remainder of this article we shall assume that $\mathcal{G}$ is a simple graph with no isolated vertices and with $s \geq 2$.

If $\mathcal{G}$ is a connected graph, the length of $C_{X}(d)$ has been determined.

Proposition 2.7 ([15, Corollary 3.8]). Let $\mathcal{G}$ be a connected graph and $X$ its associated algebraic toric set. Then $|X|=(q-1)^{n-1}$ if $\mathcal{G}$ is non-bipartite and $|X|=(q-1)^{n-2}$ if $\mathcal{G}$ is bipartite.

In particular, since $X \subset \mathbb{T}^{s-1} \subset \mathbb{P}^{s-1}$ and $\left|\mathbb{T}^{s-1}\right|=(q-1)^{s-1}$ we see that if $\mathcal{G}$ is a connected non-bipartite graph with $n=s$, then the algebraic toric set parameterized by the edges of $\mathcal{G}$ coincides with $\mathbb{T}^{s-1}$. In this situation, the vanishing ideal of $\mathbb{T}^{s-1}$, its invariants and all of the parameters of $C_{X}(d)$ are known, and are summarized in the following proposition.

Proposition 2.8. ([9, Theorem 1, Lemma 1], [17, Corollary 2.2, Theorem 3.5]) If $\mathbb{T}^{s-1}$ is the projective torus in $\mathbb{P}^{s-1}$, then

(i) $I\left(\mathbb{T}^{s-1}\right)=\left(\left\{t_{i}^{q-1}-t_{1}^{q-1}\right\}_{i=2}^{s}\right)$;

(ii) $F_{\mathbb{T}^{s-1}}(t)=\left(1-t^{q-1}\right)^{s-1} /(1-t)^{s}$;

(iii) $\operatorname{reg}\left(S / I\left(\mathbb{T}^{s-1}\right)\right)=(s-1)(q-2)$ and $\operatorname{deg}\left(S / I\left(\mathbb{T}^{s-1}\right)\right)=\left|\mathbb{T}^{s-1}\right|=(q-1)^{s-1}$;

(iv) $\operatorname{dim}_{K} C_{\mathbb{T}^{s-1}}(d)=\sum_{j=0}^{\lfloor d /(q-1)\rfloor}(-1)^{j}\left(\begin{array}{c}s-1 \\ j\end{array}\right)\left(\begin{array}{c}s-1+d-j(q-1) \\ s-1\end{array}\right)$;

(v) $\delta_{\mathbb{T}^{s-1}}(d)=(q-1)^{s-(k+2)}(q-1-\ell)$ for all $d<\operatorname{reg}\left(S / I\left(\mathbb{T}^{s-1}\right)\right)$, where $k \geq 0$ and $1 \leq \ell \leq q-2$ are the unique integers such that $d=k(q-2)+\ell$.

In the statement of the result, $F_{\mathbb{T}^{s-1}}(t)=\sum_{i=0}^{\infty} H_{\mathbb{T}^{s-1}}(i) t^{i}$ is the Hilbert series of $S / I\left(\mathbb{T}^{s-1}\right)$. The fact that the vanishing ideal in the case of the torus is a complete intersection plays a crucial part in the proof of these results. We know that in practice the vanishing ideal associated to a general graph is far from being a complete intersection. Indeed, by [17, Corollary 4.5] for an algebraic toric set $X$ associated to a graph (or more generally a clutter - see [17] for a definition), $I(X)$ is a complete intersection if and only if $X=\mathbb{T}^{s-1}$.

\section{The Length of PARAMETERIZED CODES OF GRAPHS}

We continue to use the notation and definitions used in Section 2, In this section, we show an explicit formula for the length of any parameterized code associated to an arbitrary graph.

Let $\mathcal{G}$ be a simple graph with vertex set $V_{\mathcal{G}}=\left\{v_{1}, \ldots, v_{n}\right\}$ and edge set $E_{\mathcal{G}}=\left\{e_{1}, \ldots, e_{s}\right\}$. Denote by $\mathcal{G}_{1}, \ldots, \mathcal{G}_{m}$ the connected components of $\mathcal{G}$. For each $1 \leq j \leq m$, let $n_{j}$ and $s_{j}$ denote the number of vertices and edges of $\mathcal{G}_{j}$, respectively; so that $n=n_{1}+\cdots+n_{m}$ and $s=s_{1}+\cdots+s_{m}$. Denote the edges of $\mathcal{G}_{j}$ by $\left\{e_{j 1}, \ldots, e_{j s_{j}}\right\}$, let $X_{j} \subset \mathbb{P}^{s_{j}-1}$ be the algebraic toric set parameterized by $\mathcal{G}_{j}$ and let

$$
\left(K^{*}\right)^{n_{j}} \stackrel{\theta_{j}}{\longrightarrow} X_{j}^{*} \stackrel{\tilde{\pi}_{j}}{\longrightarrow} X_{j} \longrightarrow 1
$$


be the corresponding structural sequences. Since for fixed distinct $j_{1} \neq j_{2}$ the edges $e_{j_{1} k_{1}}$ and $e_{j_{2} k_{2}}$ have no vertex in common and thus $\mathbf{x}^{e_{j_{1} k_{1}}}$ and $\mathbf{x}^{e_{j_{2} k_{2}}}$ involve disjoint sets of coordinates of the vector $\mathbf{x}$, we deduce that $\theta:\left(K^{*}\right)^{n} \rightarrow X^{*}$ is isomorphic to

$$
\theta_{1} \times \cdots \times \theta_{m}:\left(K^{*}\right)^{n_{1}} \times \cdots \times\left(K^{*}\right)^{n_{m}} \rightarrow X_{1}^{*} \times \cdots \times X_{m}^{*} .
$$

In particular $\left|X^{*}\right|=\prod_{j=1}^{m}\left|X_{j}^{*}\right|$. We need to find the order of the kernel of the maps $\tilde{\pi}_{j}$.

Lemma 3.1. Let $\mathcal{G}$ be a connected graph. If $\mathcal{G}$ is non-bipartite, then $|\operatorname{Ker} \widetilde{\pi}|=\frac{q-1}{2}$ if $q$ is odd and $|\operatorname{Ker} \widetilde{\pi}|=q-1$ if $q$ is even. If $\mathcal{G}$ is bipartite, then $|\operatorname{Ker} \widetilde{\pi}|=q-1$.

Proof. Let $\mathbf{x} \in\left(K^{*}\right)^{n}$. Then $\theta(\mathbf{x})=(1, \ldots, 1)$ implies that $\mathbf{x}^{e}=1$ for all $e \in E_{\mathcal{G}}$. Suppose $\mathcal{G}$ is non-bipartite. Then $\mathcal{G}$ contains an odd cycle. We assume, without loss of generality, that the edges in this cycle are

$$
e_{1}=\left\{v_{1}, v_{2}\right\}, \ldots, e_{2 k-1}=\left\{v_{2 k-1}, v_{1}\right\}
$$

where $v_{1} \ldots, v_{2 k-1} \in V_{\mathcal{G}}$. We deduce that $x_{1} x_{2}=\cdots=x_{2 k-1} x_{1}=1$, which, in turn, implies that $x_{1}=\cdots=x_{2 k-1}=u \in K^{*}$ with $u^{2}=1$. Now, let $v_{r} \in V_{\mathcal{G}}$ be any vertex of $\mathcal{G}$. Then, there exists a path

$$
\left\{v_{1}, v_{\ell_{1}}\right\},\left\{v_{\ell_{1}}, v_{\ell_{2}}\right\}, \ldots,\left\{v_{\ell_{k}}, v_{r}\right\}
$$

connecting $x_{1}$ with $x_{r}$. Since $x_{1} x_{j_{1}}=x_{j_{1}} x_{j_{2}}=\cdots=x_{j_{k}} x_{r}=1$, we deduce that $x_{r}=u$. Hence, either $\mathbf{x}=(1, \ldots, 1)$ or $\mathbf{x}=(-1, \ldots,-1)$, from which we conclude that $|\operatorname{Ker} \theta|=2$ if $q$ is odd and $|\operatorname{Ker} \theta|=1$ if $q$ even. Suppose now that $\mathcal{G}$ is bipartite, and, without loss of generality, denote the bipartition of $V_{\mathcal{G}}$ by $\left\{v_{1}, \ldots, v_{\ell}\right\} \cup\left\{v_{\ell+1}, \ldots, v_{n}\right\}$. Let $v_{r}$ be any vertex and let

$$
\left\{v_{1}, v_{j_{1}}\right\},\left\{v_{j_{1}}, v_{j_{2}}\right\}, \ldots,\left\{v_{j_{k}}, v_{r}\right\}
$$

be a path connecting $v_{1}$ with $v_{r}$. Notice that $\left\{v_{j_{1}}, v_{j_{3}}, \ldots\right\}$ is a subset of $\left\{v_{\ell+1}, \ldots, v_{n}\right\}$ and $\left\{v_{j_{2}}, v_{j_{4}}, \ldots\right\}$ is a subset of $\left\{v_{1}, \ldots, v_{\ell}\right\}$. From $x_{1} x_{j_{1}}=x_{j_{1}} x_{j_{2}}=\cdots=x_{j_{k}} x_{r}=1$ we deduce that $x_{r}=x_{1}$ if $v_{r} \in\left\{v_{1}, \ldots, v_{\ell}\right\}$ or $x_{r}=x_{1}^{-1}$ otherwise. Hence $x=\left(x_{1}, \ldots, x_{1}, x_{1}^{-1}, \ldots, x_{1}^{-1}\right)$, i.e., the $\ell$ first coordinates of $\mathbf{x}$ are equal to $x_{1}$ and the remaining ones are equal to $x_{1}^{-1}$. Conversely, it is easy to see that any element of $\left(K^{*}\right)^{n}$ of the form $\left(u, \ldots, u, u^{-1}, \ldots, u^{-1}\right)$ belongs to $\operatorname{Ker} \theta$. We deduce that in this case $|\operatorname{Ker} \theta|=q-1$. The proof now follows easily from Proposition 2.7. Indeed, we know that the order of $X$ is $(q-1)^{n-1}$, if $\mathcal{G}$ is non-bipartite and $(q-1)^{n-2}$ otherwise. Hence, $|\operatorname{Ker} \widetilde{\pi}|=\frac{q-1}{2}$ if $\mathcal{G}$ is non-bipartite and $q$ is odd, $|\operatorname{Ker} \widetilde{\pi}|=q-1$ if $\mathcal{G}$ is non-bipartite and $q$ is even, and $|\operatorname{Ker} \widetilde{\pi}|=q-1$ if $\mathcal{G}$ is bipartite.

We come to the main result of this section.

Theorem 3.2. Suppose $\mathcal{G}$ has $m$ connected components, of which $\gamma$ are non-bipartite. Then,

$$
|X|=\left\{\begin{array}{l}
\left(\frac{1}{2}\right)^{\gamma-1}(q-1)^{n-m+\gamma-1}, \text { if } \gamma \geq 1 \text { and } q \text { is odd } \\
(q-1)^{n-m+\gamma-1}, \text { if } \gamma \geq 1 \text { and } q \text { is even, } \\
(q-1)^{n-m-1}, \text { if } \gamma=0 .
\end{array}\right.
$$

Proof. As in the discussion above, let $X_{1}, \ldots, X_{m}$ be the parameterized toric sets associated to the connected components of $\mathcal{G}$. Then $\left|X^{*}\right|=\prod_{j=1}^{m}\left|X_{j}^{*}\right|$, which, by Lemma 3.1, is given by

$$
\left|X^{*}\right|=\left\{\begin{array}{l}
\left(\frac{1}{2}\right)^{\gamma}(q-1)^{n-m+\gamma}, \text { if } q \text { is odd, } \\
(q-1)^{n-m+\gamma}, \text { if } q \text { is even. }
\end{array}\right.
$$

From the proof of Lemma 3.1, it is seen that the kernel of the map $\tilde{\pi}: X^{*} \rightarrow X$ is equal to $\Lambda$, the diagonal subgroup of $\left(K^{*}\right)^{s}$, if $\gamma=0$, and it is equal to $\Lambda^{2}=\left\{\left(\lambda^{2}, \ldots, \lambda^{2}\right) \mid \lambda \in F_{q}^{*}\right\}$ if $\gamma \geq 1$. 
The subgroup $\Lambda$ has order $q-1$. The subgroup $\Lambda^{2}$ has order $q-1$ if $q$ is even and has order $(q-1) / 2$ if $q$ is odd (this follows readily using the map $\left.\lambda \mapsto\left(\lambda^{2}, \ldots, \lambda^{2}\right)\right)$. As $|X|=\left|X^{*}\right| /|\operatorname{Ker} \widetilde{\pi}|$, the result follows.

Example 3.3. Let $G$ be the graph whose connected components are a triangle and a square. Thus, $n=7, m=2, \gamma=1$. Using the formula of Theorem 3.2, we get: (a) $|X|=1024$ if $q=5$, and (b) $|X|=243$ if $q=2^{2}$.

\section{Degree bounds For the Generators of $I(X)$}

We continue to use the notation and definitions used in Section 2, In this section $X \subset \mathbb{P}^{s-1}$ is the algebraic toric set parameterized by $\nu_{1}, \ldots, \nu_{s} \in \mathbb{N}^{n}$ and $I(X) \subset S=K\left[t_{1}, \ldots, t_{s}\right]$ is the vanishing ideal of $X$. We show some degree bounds for a minimal set of generators of $I(X)$ consisting of binomials.

Recall that by [15] we know that $I(X)$ is generated by homogeneous binomials $t^{a}-t^{b}$, with $a, b \in \mathbb{N}^{s}$. There are a number of elementary observations to be made. Let $f=t^{a}-t^{b}$ be a nonzero binomial of $S$. Firstly, since $X \subset \mathbb{T}^{s-1}$, evidently $I\left(\mathbb{T}^{s-1}\right) \subset I(X)$, hence $t_{i}^{q-1}-t_{j}^{q-1} \in I(X)$, for all $1 \leq i, j \leq s$. Secondly, if $\operatorname{gcd}\left(t^{a}, t^{b}\right) \neq 1$, then we can factor the greatest common divisor $t^{c}$ from both $t^{a}$ and $t^{b}$ to obtain $t^{a}-t^{b}=t^{c}\left(t^{a^{\prime}}-t^{b^{\prime}}\right)$, for some $a^{\prime}, b^{\prime} \in \mathbb{N}^{s}$. Since $t^{c}$ is never zero on $\mathbb{T}^{s-1}$, for any $c \in \mathbb{N}^{s}$, we deduce that $t^{a}-t^{b} \in I(X)$ if and only if $t^{a^{\prime}}-t^{b^{\prime}} \in I(X)$. Therefore, when looking for "binomial generators" of $I(X)$ we may restrict ourselves to those binomials $t^{a}-t^{b}$ such that $t^{a}$ and $t^{b}$ have no common divisors. Given $a=\left(a_{1}, \ldots, a_{s}\right) \in \mathbb{N}^{s}$, we set $|a|=a_{1}+\cdots+a_{s}$ and $\operatorname{supp}(a)=\left\{i: a_{i} \neq 0\right\}$. Then, clearly, $t^{a}$ and $t^{b}$ have no common divisors if and only if $\operatorname{supp}(a) \cap \operatorname{supp}(b)=\emptyset$.

Definition 4.1. A subgroup of $\mathbb{Z}^{s}$ is called a lattice. A lattice ideal is an ideal of the form

$$
I(\mathcal{L})=\left(\left\{t^{a}-t^{b}: a-b \in \mathcal{L} \text { and } \operatorname{supp}(a) \cap \operatorname{supp}(b)=\emptyset\right\}\right) \subset S
$$

for some lattice $\mathcal{L}$ of $\mathbb{Z}^{s}$.

Lemma 4.2. Let $L \subset S$ be a lattice ideal generated by $\mathcal{B}=\left\{t^{a_{i}}-t^{b_{i}}\right\}_{i=1}^{r}$. Then, (a) $L=I(\mathcal{L})$, where $\mathcal{L}$ is the subgroup of $\mathbb{Z}^{s}$ generated by $\left\{a_{i}-b_{i}\right\}_{i=1}^{r}$, and (b) if $t^{a_{i}}-t^{b_{i}}$ is homogeneous for all $i$ and $f=t^{a}-t^{b} \in L$, then $f$ is homogeneous.

Proof. Part (a) follows from [14, Lemma 7.6]. To show (b) notice that, from part (a), $f \in I(\mathcal{L})$. Then, $a-b$ is a linear combination of $\left\{a_{i}-b_{i}\right\}_{i=1}^{r}$. Thus, if $\mathbf{1}=(1, \ldots, 1)$, we get that $|a|-|b|$ is equal to $\langle\mathbf{1}, a-b\rangle=0$ because $\left\langle\mathbf{1}, a_{i}-b_{i}\right\rangle=0$ for all $i$. Thus, $|a|=\operatorname{deg}\left(t^{a}\right)=\operatorname{deg}\left(t^{b}\right)=|b|$.

Lemma 4.3. If $f=t^{a}-t^{b} \in I(X)$, then $f$ is homogeneous.

Proof. According to [15, Theorem 2.1], $I(X)$ is lattice ideal generated by homogeneous binomials. Thus, the lemma follows from Lemma 4.2.

Lemma 4.4. Let $f=t^{a}-t^{b} \in I(X)$, where $a, b \in \mathbb{N}^{s}$ and $\operatorname{supp}(a) \cap \operatorname{supp}(b)=\emptyset$. Suppose that there exists $i$ such that $t_{i}^{q-1}$ divides $t^{a}$ and $\operatorname{supp}(b) \neq \emptyset$. Then, there exists a binomial $g \in I(X)$, with $\operatorname{deg}(g)<\operatorname{deg}(f)$, and there exists $j$, such that $f-t_{j} g \in I\left(\mathbb{T}^{s-1}\right)$.

Proof. Write $t^{a}=t_{i}^{q-1} t^{a^{\prime}}$, with $a^{\prime} \in \mathbb{N}^{s}$. Since $\operatorname{supp}(b) \neq \emptyset$, there exists $j$ such that $t_{j}$ divides $t^{b}$. Write $t^{b}=t_{j} t^{b^{\prime}}$, for some $b^{\prime} \in \mathbb{N}^{s}$. Then,

$$
t^{a}-t^{b}=t_{i}^{q-1} t^{a^{\prime}}-t_{j} t^{b^{\prime}}=\left(t_{i}^{q-1}-t_{j}^{q-1}\right) t^{a^{\prime}}+t_{j}\left(t_{j}^{q-2} t^{a^{\prime}}-t^{b^{\prime}}\right) .
$$


Set $g=t_{j}^{q-2} t^{a^{\prime}}-t^{b^{\prime}}$. Then, since $t_{i}^{q-1}-t_{j}^{q-1} \in I(X)$, we see that $g \in I(X)$ and, moreover, it is clear that if $g \neq 0$ then $\operatorname{deg}(g)=\operatorname{deg}(f)-1$.

Theorem 4.5. There exists a set of generators of $I(X)$ which consists of the toric relations $t_{i}^{q-1}-t_{j}^{q-1}$ plus a finite set of homogeneous binomials $t^{a}-t^{b}$ with $\operatorname{supp}(a) \cap \operatorname{supp}(b)=\emptyset$ and such that the degree of $t^{a}-t^{b}$ in each of the variables $t_{i}$ is $\leq q-2$.

Proof. We know that $I(X)$ is generated by binomials [15]. If $\left\{f_{1}, \ldots, f_{r}\right\}$ is a set of binomials generating $I(X)$, then so is the set

$$
\mathcal{B}=\left\{f_{1}, \ldots, f_{r}\right\} \cup\left\{t_{i}^{q-1}-t_{j}^{q-1}: 1 \leq i, j \leq s\right\} .
$$

If $f_{i} \in I\left(\mathbb{T}^{s-1}\right)$, we have $(\mathcal{B})=\left(\left\{\left\{f_{1}, \ldots, f_{r}\right\} \backslash\left\{f_{i}\right\}\right\} \cup\left\{t_{i}^{q-1}-t_{j}^{q-1}: 1 \leq i, j \leq s\right\}\right)$. Thus, we may assume that $\mathcal{B}$ is a generating set of $I(X)$ with $f_{i} \notin I\left(\mathbb{T}^{s-1}\right)$ for all $i$. By the discussion above we may also assume that each $f_{i}$ is of the form $t^{a}-t^{b}$ with $\operatorname{supp}(a) \cap \operatorname{supp}(b)=\emptyset$. We can write $f_{1}=t^{a}-t^{b}$, with $a, b \in \mathbb{N}^{s}$. Suppose that there exists $i$ such that $t_{i}^{q-1}$ divides $t^{a}$ or $t^{b}$. Hence, since $f_{1}$ is homogeneous by Lemma 4.3, we deduce that the sets $\operatorname{supp}(a)$ and $\operatorname{supp}(b)$ are both nonempty. Then, from Lemma 4.4, there exists $j$ and a homogeneous binomial $g_{1}^{\prime} \in I(X)$ such that $\operatorname{deg}\left(g_{1}^{\prime}\right)<\operatorname{deg}\left(f_{1}\right)$ and $f_{1}-t_{j} g_{1}^{\prime} \in I\left(\mathbb{T}^{s-1}\right)$. We can write $g_{1}^{\prime}=t^{c} g_{1}$ for some $c \in \mathbb{N}^{s}$, where $g_{1}$ is a binomial in $I(X)$ whose terms have disjoint support. Clearly,

$$
I(X)=(\mathcal{B})=\left(\left\{g_{1}, f_{2}, \ldots, f_{r}\right\} \cup\left\{t_{i}^{q-1}-t_{j}^{q-1}: 1 \leq i, j \leq s\right\}\right)
$$

and $g_{1} \notin I\left(\mathbb{T}^{s-1}\right)$. If there exists $i$ such that $t_{i}^{q-1}$ divides one of the terms of $g_{1}$, we repeat the previous procedure with $g_{1}$ playing the role of $f_{1}$ and obtain a binomial $g_{2}$, and so on. Thus, by iterating the previous procedure, we obtain a sequence of homogeneous binomials $f_{1}, g_{1}, \ldots, g_{m}$, with decreasing degrees, such that

$$
I(X)=(\mathcal{B})=\left(\left\{g_{m}, f_{2}, \ldots, f_{r}\right\} \cup\left\{t_{i}^{q-1}-t_{j}^{q-1}: 1 \leq i, j \leq s\right\}\right)
$$

and $g_{m} \notin I\left(\mathbb{T}^{s-1}\right)$. Thus, using the previous procedure enough times, we obtain a binomial $g_{m}=t^{a^{\prime}}-t^{b^{\prime}}$ none of whose terms $t^{a^{\prime}}$ or $t^{b^{\prime}}$ is divisible by any $t_{i}^{q-1}$, for $1 \leq i \leq s$. If we proceed in this manner, with each of the remaining $f_{2}, \ldots, f_{r}$, we reach a generating set satisfying the condition in the statement.

The next proposition is intended mainly for practical applications. It gives a bound on the degrees of a minimal set of generators of $I(X)$. It is a valuable tool to use when implementing the calculation of $I(X)$ in a computer algebra software.

Proposition 4.6. Set $k=\left\lfloor\frac{s}{2}\right\rfloor$. If $k \geq 2$, then the vanishing ideal of $X$ has a generating set whose elements have degree $\leq k(q-2)$.

Proof. Let $t^{a}-t^{b} \in I(X)$ be a homogeneous binomial. Write $a=\left(a_{1}, \ldots, a_{s}\right) \in \mathbb{N}^{s}$ and $b=\left(b_{1}, \ldots, b_{s}\right) \in \mathbb{N}^{s}$. By Theorem 4.5, we may assume that $\operatorname{supp}(a) \cap \operatorname{supp}(b)=\emptyset$ and that $0 \leq a_{i}, b_{j} \leq q-2$. Let $r=|\operatorname{supp}(a)|$ and $\ell=|\operatorname{supp}(b)|$. Then, either $r$ or $\ell$ is $\leq k$, for otherwise:

$$
r+\ell \geq 2 k+2=2\lfloor s / 2\rfloor+2 \geq s+1,
$$

which is impossible. Assume $r \leq k$. Then, $\operatorname{deg}\left(t^{a}-t^{b}\right)=a_{1}+\cdots+a_{s} \leq r(q-2) \leq k(q-2)$.

If $X$ is the algebraic toric set associated to a cycle $\mathcal{G}$ of order $s=2 k$, then, by Corollary 5.12 , $I(X)$ is generated in degrees $\leq(k-1)(q-2)+1$. Hence for this restricted class of vanishing ideals our estimate is not sharp. On the other hand, for $q=3$, the estimate that $I(X)$ is generated in degrees $\leq k$ is sharp, as the following example shows. 

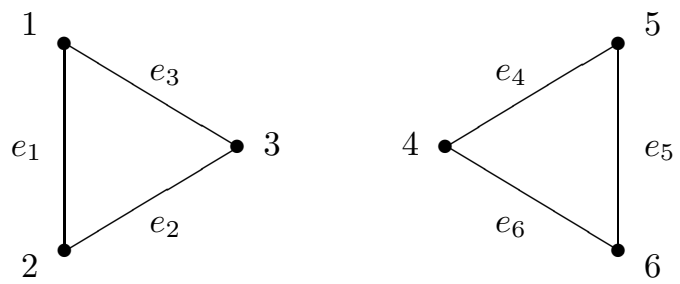

FiguRE 1.

Example 4.7. Let $\mathcal{G}$ be the graph in Figure 1 and assume that $q=3$. Then, using Macaulay 2 [12], we found that $I(X)$ is generated by the (minimal) set of binomials:

$$
\begin{array}{ccccc}
t_{5}^{2}-t_{6}^{2}, \quad t_{4}^{2}-t_{6}^{2}, \quad t_{3}^{2}-t_{6}^{2}, \quad t_{2}^{2}-t_{6}^{2}, \quad t_{1}^{2}-t_{6}^{2}, \\
t_{3} t_{4} t_{5}-t_{1} t_{2} t_{6}, \quad t_{2} t_{4} t_{5}-t_{1} t_{3} t_{6}, \quad t_{1} t_{4} t_{5}-t_{2} t_{3} t_{6}, \quad t_{2} t_{3} t_{5}-t_{1} t_{4} t_{6}, & t_{1} t_{3} t_{5}-t_{2} t_{4} t_{6}, \\
t_{1} t_{2} t_{5}-t_{3} t_{4} t_{6}, & t_{2} t_{3} t_{4}-t_{1} t_{5} t_{6}, \quad t_{1} t_{3} t_{4}-t_{2} t_{5} t_{6}, & t_{1} t_{2} t_{4}-t_{3} t_{5} t_{6}, & t_{1} t_{2} t_{3}-t_{4} t_{5} t_{6} .
\end{array}
$$

\section{Generators of $I(X)$ FOR Even CyCles and CERTAin Bipartite Graphs}

We keep the notation of Section 3: $X \subset \mathbb{P}^{s-1}$ is the algebraic toric set parameterized by a graph $\mathcal{G}$ and $I(X) \subset S=K\left[t_{1}, \ldots, t_{s}\right]$ is the vanishing ideal of $X$. This section is devoted to giving an explicit description of a binomial generating set for $I(X)$, when $\mathcal{G}=\mathcal{C}_{2 k}$ is a cycle of even order, or when $\mathcal{G}$ is a bipartite graph whose cycles are pairwise vertex disjoint.

Proposition 5.1. Let $f=t^{a}-t^{b} \in I(X)$, with $a=\left(a_{1}, \ldots, a_{s}\right)$ and $b=\left(b_{1}, \ldots, b_{s}\right)$, such that $\operatorname{supp}(a) \cap \operatorname{supp}(b)=\emptyset$ and $a_{j}, b_{j} \leq q-2$ for all $j$. (a) If $G$ is a connected bipartite graph and $e_{i}$ is an edge of $\mathcal{G}$ which does not belong to any cycle of $\mathcal{G}$, then $a_{i}=b_{i}=0$. (b) If $\mathcal{G}$ is any graph and $\mathcal{G}$ has an edge $e_{i}$ with a degree 1 incident vertex, then $a_{i}=b_{i}=0$.

Proof. (a) Assume, without loss of generality that, $e_{i}=\left\{v_{1}, v_{2}\right\}$. In what follows we use the symbol $\sqcup$ to denote a disjoint union of objects. Since $\mathcal{G}$ is bipartite there exist a bipartition $V_{G}=A \sqcup B$ with, say, $v_{1} \in A$ and $v_{2} \in B$. Since $e_{i}$ does not belong to a cycle of $\mathcal{G}$, the removal of edge $e_{i}$ produces a disconnected graph $\mathcal{G}_{1} \sqcup \mathcal{G}_{2}$, with $v_{1} \in V_{\mathcal{G}_{1}}$ and $v_{2} \in V_{\mathcal{G}_{2}}$. Let $u \in K^{*}$ be a generator of the multiplicative group of $K$. Let us label the vertices of $\mathcal{G}$ with one of the elements $u, u^{-1}$ or 1 , according to the rule that we now explain. Let $v_{r}$ be any vertex. If $v_{r} \in V_{\mathcal{G}_{1}}$ label $v_{r}$ with 1 , if $v_{r} \in V_{\mathcal{G}_{2}} \cap A$ label $v_{r}$ with $u^{-1}$, and if $v_{r} \in V_{\mathcal{G}_{2}} \cap B$ label $v_{r}$ with $u$. Consider $\mathbf{x}=\left(x_{1}, \ldots, x_{n}\right) \in\left(K^{*}\right)^{n}$ where, for $1 \leq r \leq n$, the coordinate $x_{r}$ takes on the value of the label of $v_{r}$. Then $\mathbf{x}^{e_{j}}=1$ if $j \neq i$ and $\mathbf{x}^{e_{i}}=u$. Assume that $a_{i}>0$, then $b_{i}=0$ because $a$ and $b$ have disjoint support. Thus $f\left(\mathbf{x}^{e_{1}}, \ldots, \mathbf{x}^{e_{s}}\right)=0$, implies that $u^{a_{i}}-1=0$, a contradiction because $1 \leq a_{i} \leq q-2$. Similarly if $b_{i}>0$ we derive a contradiction. Hence, we deduce that $a_{i}=b_{i}=0$. (b) This part follows using a similar argument.

Example 5.2. For non-bipartite graphs Proposition 5.1(a) does not hold. Let $\mathcal{G}$ be the graph in Figure 2 and assume that $q=5$. Then, using Macaulay2 12, we found that the binomial $t_{1} t_{2} t_{4}^{2} t_{7}-t_{3} t_{5}^{2} t_{6} t_{8}$ is in a minimal generating set of $I(X)$. In this monomial the variables $t_{4}$ and $t_{5}$, which are not in any cycle of $\mathcal{G}$, occur.

Corollary 5.3. Suppose that $\mathcal{G}=\mathcal{C}_{2 k}$ is a cycle of even order. Let $f=t^{a}-t^{b}$ be a nonzero homogeneous binomial in $I(X)$, with $a=\left(a_{1}, \ldots, a_{s}\right) \in \mathbb{N}^{s}$ and $b=\left(b_{1}, \ldots, b_{s}\right) \in \mathbb{N}^{s}$ such that $\operatorname{supp}(a) \cap \operatorname{supp}(b)=\emptyset$ and $0 \leq a_{i}, b_{j} \leq q-2$. Then $\operatorname{supp}(a) \cup \operatorname{supp}(b)=\{1, \ldots, s\}$. 


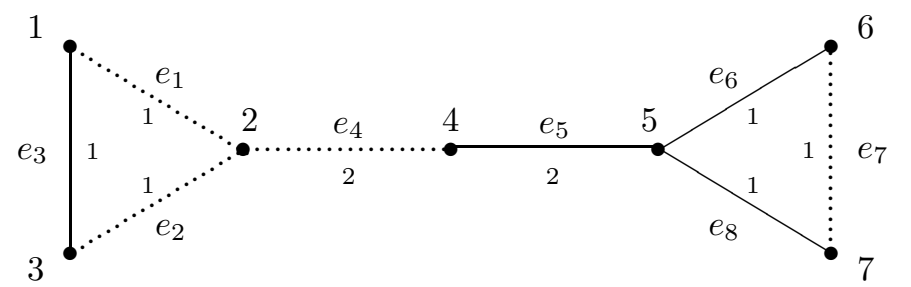

Figure 2.

Proof. Assume, without loss of generality that $s \notin \operatorname{supp}(a) \cup \operatorname{supp}(b)$. Then, $f$ is a polynomial in the variables $t_{1}, \ldots, t_{s-1}$ which vanishes along the projection of $X$ onto the first $s-1$ coordinates. The algebraic toric set obtained after projecting is none other that the algebraic toric set associated with the graph obtained from $\mathcal{G}=\mathcal{C}_{2 k}$ by removing the edge $e_{s}$, which is a tree. Hence, by Proposition [5.1, none of the remaining variables $t_{1}, \ldots, t_{s-1}$ occurs in $f$, in other words, $f=0$, which is a contradiction.

From now on, until otherwise stated, we will restrict to the case of $\mathcal{G}=\mathcal{C}_{2 k}$, a cycle of order $2 k$ with $k \geq 2$. Let $V_{\mathcal{C}_{2 k}}=\left\{v_{1}, \ldots, v_{2 k}\right\}$ and $e_{i}=\left\{v_{i}, v_{i+1}\right\}$ for $1 \leq i \leq 2 k-1$ and $e_{s}=e_{2 k}=\left\{v_{2 k}, v_{1}\right\}$. We are now ready to give a combinatorial description of the generators of $I(X)$ other than those coming from the toric relations. From Theorem 4.5 and Corollary 5.3 we know that there is a set of generators of $I(X)$ consisting of the toric generators $t_{i}^{q-1}-t_{j}^{q-1}$ plus a set of binomials of the type $t^{a}-t^{b}$ where $a=\left(a_{1}, \ldots, a_{s}\right) \in \mathbb{N}^{s}, b=\left(b_{1}, \ldots, b_{s}\right) \in \mathbb{N}^{s}$ are such that $\operatorname{supp}(a) \sqcup \operatorname{supp}(b)=\{1, \ldots, s\}$ and $0 \leq a_{i}, b_{j} \leq q-2$. Hence to any such binomial one can associate a partition of $\{1, \ldots, s\}$. For the remainder of this article, given $r \in\{1, \ldots, q-2\}$ we will fix the following notation:

$$
\hat{r}=q-1-r .
$$

Definition 5.4. Let $\sigma=A \sqcup B$ be a partition of $\{1, \ldots, s\}$ and fix $r \in\{1, \ldots, q-2\}$. Define a function $\rho_{\sigma}^{r}:\{1, \ldots, s\} \rightarrow\{r, \hat{r}\}$, recursively, by setting $\rho_{\sigma}^{r}(1)=r$ and,

$$
\begin{cases}\rho_{\sigma}^{r}(i+1)=\widehat{\rho_{\sigma}^{r}(i)}, & \text { if }\{i, i+1\} \subset A \text { or }\{i, i+1\} \subset B \\ \rho_{\sigma}^{r}(i+1)=\rho_{\sigma}^{r}(i), & \text { otherwise, }\end{cases}
$$

for every $1 \leq i \leq s-1$.

Notice that, for every $i \in\{1, \ldots, s-2\}, \rho_{\sigma}^{r}(i)=\rho_{\sigma}^{r}(i+2)$ if and only if $i$ and $i+2$ are in the same partition. Since $s$ is even, we deduce that $\rho_{\sigma}^{r}(1)=\rho_{\sigma}^{r}(s-1)$ if and only if 1 and $s-1$ are in the same partition. This implies that $\rho_{\sigma}^{r}(1)$ can be defined from $\rho_{\sigma}^{r}(s)$ using the same recursive formula. Indeed, working in $\{1, \ldots, s\}$ modulo $s$, the function $\rho_{\sigma}^{r}$ can be recovered recursively, using the above rule, from $\rho_{\sigma}^{r}(k)$, for any $k \in\{1, \ldots, s\}$. The following lemma will be used in the proofs of some of the results below.

Lemma 5.5. Let $\sigma=A \sqcup B$ be a partition of $\{1, \ldots, s\}$ and $r \in\{1, \ldots, q-2\}$. Consider $i \in A$ and $\sigma^{\prime}=A^{\prime} \sqcup B^{\prime}$ where $A^{\prime}=A \backslash\{i\}$ and $B^{\prime}=B \cup\{i\}$. Let $\rho:\{1, \ldots, s\} \rightarrow\{r, \hat{r}\}$ be given by $\rho(j)=\rho_{\sigma}^{r}(j)$ for every $j \neq i$ and $\rho(i)=\widehat{\rho_{\sigma}^{r}(i)}$. Then $\rho=\rho_{\sigma^{\prime}}^{r}$, if $i>1$ or $\rho=\rho_{\sigma^{\prime}}^{\hat{r}}$, if $i=1$.

Proof. We will look first at the case $i=1$. In this case, $\rho(1)=\widehat{\rho_{\sigma}^{r}(1)}=\hat{r}=\rho_{\sigma^{\prime}}^{\hat{r}}(1)$. If $2 \in A$, then $\rho(2)=\rho_{\sigma}^{r}(2)=\hat{r}$, according to the definition of the function $\rho$, to Definition 5.4 and to the fact that $1 \in A$. But if $2 \in A$, then $2 \in A^{\prime}$, and $\rho_{\sigma^{\prime}}^{\hat{r}}(2)=\hat{r}$ since $1 \in B^{\prime}$. If $2 \in B$, then $\rho(2)=\rho_{\sigma}^{r}(2)=r$; but if $2 \in B$, then $2 \in B^{\prime}$, and $\rho_{\sigma^{\prime}}^{\hat{r}}(2)=r$. In any case, $\rho(2)=\rho_{\sigma^{\prime}}^{\hat{r}}(2)$. Let 
$j \in\{3, \ldots, s\}$. By definition, $\rho(j)=\rho_{\sigma}^{r}(j)$; and $\rho_{\sigma}^{r}(j)$ is determined by $\sigma$, by $\rho_{\sigma}^{r}(2)$ and by Eq. (5.1). Since $\rho_{\sigma^{\prime}}^{\hat{r}}(j)$ is determined by $\sigma^{\prime}$, by $\rho_{\sigma^{\prime}}^{\hat{r}}(2)$ and by Eq. (5.1), since $\rho_{\sigma}^{r}(2)=\rho_{\sigma^{\prime}}^{\hat{r}}(2)$, and since the partitions $\sigma$ and $\sigma^{\prime}$ agree in $\{2, \ldots, s\}$, we conclude that $\rho(j)=\rho_{\sigma}^{r}(j)=\rho_{\sigma^{\prime}}^{\hat{r}}(j)$. Therefore, $\rho=\rho_{\sigma^{\prime}}^{\hat{r}}$. For the case $i>1$, we use a similar argument to show that $\rho=\rho_{\sigma^{\prime}}^{r}$.

Given any $\sigma=A \sqcup B$, a partition of $\{1, \ldots, s\}$, if, without loss in generality, we choose $1 \in A$, it is clear that given any $r \in\{1, \ldots, q-2\}$, there exist unique $a$ and $b$ in $\mathbb{N}^{s}$ such that $\operatorname{supp}(a)=A, \operatorname{supp}(b)=B, a_{i}=\rho_{\sigma}^{r}(i)$ if $i \in \operatorname{supp}(a)$ and $b_{j}=\rho_{\sigma}^{r}(j)$ if $j \in \operatorname{supp}(b)$.

Definition 5.6. Let $\sigma=A \sqcup B$ be a partition of $\{1, \ldots, s\}$ with $1 \in A$ and let $r \in\{1, \ldots, q-2\}$. We denote by $f_{\sigma}^{r}$ the unique binomial $t^{a}-t^{b} \operatorname{such}$ that $\operatorname{supp}(a)=A, \operatorname{supp}(b)=B, a_{i}=\rho_{\sigma}^{r}(i)$ if $i \in \operatorname{supp}(a)$ and $b_{j}=\rho_{\sigma}^{r}(j)$ if $j \in \operatorname{supp}(b)$.

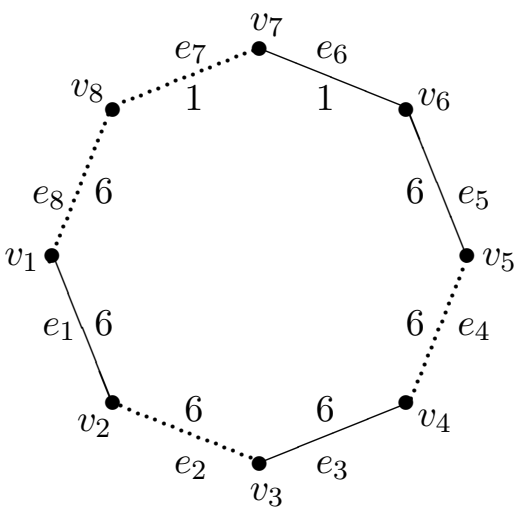

FiguRE 3.

The combinatorial data that give rise to a binomial $f_{\sigma}^{r}=t^{a}-t^{b}$ is clarified by representing it in the graph $\mathcal{G}$, by putting a label $r$ or $\widehat{r}$ to each edge. Figure 3 illustrates the map $\rho_{\sigma}^{6}$ when $q=8, r=6, s=8$ and $\sigma=\{1,3,5,6\} \sqcup\{2,4,7,8\}$. The labels of the edges correspond to the exponents of the variables in the corresponding binomial of $I(X)$. Thus, $f_{\sigma}^{6}=t_{1}^{6} t_{3}^{6} t_{5}^{6} t_{6}-t_{2}^{6} t_{4}^{6} t_{7} t_{8}^{6}$.

Lemma 5.7. Let $\sigma=A \sqcup B$ be a partition of $\{1, \ldots, s\}$ and let $r \in\{1, \ldots, q-2\}$. Suppose that $1 \in A$ and that there exists $i \in A$ such that $i>2$ and $i-1 \notin A$. Let $\sigma^{\prime}$ be the partition given by $A^{\prime} \sqcup B^{\prime}$ where $A^{\prime}=(A \backslash\{i\}) \cup\{i-1\}$ and $B^{\prime}=(B \backslash\{i-1\}) \cup\{i\}$. Then $f_{\sigma}^{r} \in I(X)$ if and only if $f_{\sigma^{\prime}}^{r} \in I(X)$.

Proof. Let $f_{\sigma}^{r}=t^{a}-t^{b}$. Using the assumption, we can write $t^{a}=t_{i}^{c} t^{a^{\prime}}$ and $t^{b}=t_{i-1}^{c} t^{b^{\prime}}$, where $c=a_{i}=b_{i-1}$ and $a^{\prime}, b^{\prime} \in \mathbb{N}^{s}$. Then:

$$
\begin{aligned}
\left(t_{i-1} t_{i}\right)^{\hat{c}} f_{\sigma}^{r} & =t_{i-1}^{\hat{c}} t_{i}^{q-1} t^{a^{\prime}}-t_{i-1}^{q-1} t_{i}^{\hat{c}} t^{b^{\prime}} \\
& =t_{i-1}^{\hat{c}} t_{i}^{q-1} t^{a^{\prime}}-t_{i-1}^{\hat{c}} t_{i-1}^{q-1} t^{a^{\prime}}+t_{i-1}^{\hat{c}} t_{i-1}^{q-1} t^{a^{\prime}}-t_{i-1}^{q-1} t_{i}^{\hat{c}} t^{b^{\prime}} \\
& =t_{i-1}^{\hat{c}} t^{a^{\prime}}\left(t_{i}^{q-1}-t_{i-1}^{q-1}\right)+\left(t_{i-1}^{\hat{c}} t^{a^{\prime}}-t_{i}^{\hat{c}} t^{b^{\prime}}\right) t_{i-1}^{q-1} .
\end{aligned}
$$

Since $t_{j}$ is never zero on $X$ we get:

$$
f_{\sigma}^{r} \in I(X) \Leftrightarrow\left(t_{i-1} t_{i}\right)^{\hat{c}} f_{\sigma}^{r} \in I(X) \Leftrightarrow\left(t_{i-1}^{\hat{c}} t^{a^{\prime}}-t_{i}^{\hat{c}} t^{b^{\prime}}\right) t_{i-1}^{q-1} \in I(X) \Leftrightarrow t_{i-1}^{\hat{c}} t^{a^{\prime}}-t_{i}^{\hat{c}} t^{b^{\prime}} \in I(X) .
$$

Now let $a^{\sharp}, b^{\sharp} \in \mathbb{N}^{s}$ be such that $t^{a^{\sharp}}=t_{i-1}^{\hat{c}} t^{a^{\prime}}$ and $t^{b^{\sharp}}=t_{i}^{\hat{c}} t^{b^{\prime}}$. Then, $\sigma^{\prime}=\operatorname{supp}\left(a^{\sharp}\right) \sqcup \operatorname{supp}\left(b^{\sharp}\right)$ is the partition of $\{1, \ldots, s\}$ obtained from interchanging $i-1$ and $i$ in $A \sqcup B$. Applying Lemma 5.5 twice, we deduce that $f_{\sigma^{\prime}}^{r}=t_{i-1}^{\hat{c}} t^{a^{\prime}}-t_{i}^{\hat{c}} t^{b^{\prime}}$. 
Lemma 5.8. Let $\sigma=A \sqcup B$ be a partition of $\{1, \ldots, s\}$ with $1 \in A$ and let $r \in\{1, \ldots, q-2\}$. If $f_{\sigma}^{r} \in I(X)$ then $|A|=|B|$.

Proof. Let $\ell=|A|$. Using sufficiently many times Lemma 5.7, we may assume that $\sigma$ is the partition $\{1, \ldots, \ell\} \sqcup\{\ell+1, \ldots, s\}$. Accordingly,

$$
f_{\sigma}^{r}=t_{1}^{r} t_{2}^{\hat{r}} \cdots t_{\ell}^{r^{\prime}}-t_{\ell+1}^{r^{\prime}} \cdots t_{s-1}^{\hat{r}} t_{s}^{r}
$$

where $r^{\prime} \in\{r, \hat{r}\}$. Now $\operatorname{deg}\left(t_{j}^{r} \cdots\right)$, for a monomial consisting of a product of variables with consecutive exponents alternating in $\{r, \hat{r}\}$, is a strictly increasing function with respect to the number of variables involved. Since $f_{\sigma}^{r}$ is homogeneous (by Lemma 4.3) we deduce that $|A|=\ell=s-\ell=|B|$.

We come to one of the main results of this section, a combinatorial description of a generating set for a vanishing ideals over an even cycle.

Theorem 5.9. Let $I(X)$ be the vanishing ideal of the algebraic toric set $X$ associated to an even cycle $\mathcal{G}=\mathcal{C}_{2 k}$. Then, $I(X)$ is generated by the binomials $t_{i}^{q-1}-t_{j}^{q-1}, 1 \leq i, j \leq s=2 k$, and the binomials $f_{\sigma}^{r}$ obtained from all $r \in\{1, \ldots, q-2\}$ and all partitions $\sigma=A \sqcup B$ of $\{1, \ldots, s\}$ with $|A|=|B|$.

Proof. By Theorem 4.5 and Corollary 5.3, we know that $I(X)$ is generated by the binomials of the form $t_{i}^{q-1}-t_{j}^{q-1}, 1 \leq i, j \leq s=2 k$, and the homogeneous binomials $f=t^{a}-t^{b}$ with $a=\left(a_{1}, \ldots, a_{s}\right) \in \mathbb{N}^{s}$ and $b=\left(b_{1}, \ldots, b_{s}\right) \in \mathbb{N}^{s}$ such that $\operatorname{supp}(a) \sqcup \operatorname{supp}(b)=\{1, \ldots, s\}$ and $0 \leq a_{i}, b_{j} \leq q-2$. Let $f$ be a binomial of the latter type. We may assume that $1 \in \operatorname{supp}(a)$, for we can always replace $f$ by $-f$ in a generating set of $I(X)$. Set $\sigma=\operatorname{supp}(a) \sqcup \operatorname{supp}(b)$ and let $r=a_{1}$. Let us show that $f=f_{\sigma}^{r}$, i.e., let us show that $a_{i}=\rho_{\sigma}^{r}(i)$, for every $i \in \operatorname{supp}(a) \backslash\{1\}$ and $b_{j}=\rho_{\sigma}^{r}(j)$ for every $j \in \operatorname{supp}(b)$. Let $i \in \operatorname{supp}(a) \backslash\{1\}$ and let $u \in K^{*}$ be a generator of the multiplicative group of $K$. Consider $\mathbf{x} \in\left(K^{*}\right)^{n}$ given by setting $x_{i}=u$ and $x_{j}=1$ for all $j \neq i$. Then, $f\left(\mathbf{x}^{\nu_{1}}, \ldots, \mathbf{x}^{\nu_{s}}\right)=0$ implies that $u^{a_{i-1}} u^{a_{i}}=1$, if $i-1 \in \operatorname{supp}(a)$ or $u^{a_{i}}=u^{b_{i-1}}$ if $i-1 \in \operatorname{supp}(b)$. We get, in the first case, $a_{i}=q-1-a_{i-1}=\rho_{\sigma}^{r}(i)$, and, in the second case, $a_{i}=b_{i-1}=\rho_{\sigma}^{r}(i)$. Similarly, if $j \in \operatorname{supp}(b)$, then $b_{j}=\rho_{\sigma}^{r}(j)$. Since $f_{\sigma}^{r} \in I(X)$, by Lemma 5.8, $|A|=|B|$.

To complete the proof let $\sigma=A \sqcup B$ be a partition of $\{1, \ldots, s\}$ with $|A|=|B|, r \in\{1, \ldots, q-2\}$ and let us show that $f_{\sigma}^{r} \in I(X)$. By Lemma 5.7, we may assume that $\sigma$ is the partition $\{1, \ldots, k\} \sqcup\{k+1, \ldots, s\}$ and $f_{\sigma}^{r}=t_{1}^{r} t_{2}^{\hat{r}} \cdots t_{k}^{r^{\prime}}-t_{k+1}^{r^{\prime}} \cdots t_{s-1}^{\hat{r}} t_{s}^{r}$, where $r^{\prime} \in\{r, \hat{r}\}$. Now, let $\mathbf{x} \in\left(K^{*}\right)^{n}$. Then $f_{\sigma}^{r}\left(\mathbf{x}^{\nu_{1}}, \ldots, \mathbf{x}^{\nu_{s}}\right)=x_{1}^{r} x_{k+1}^{r^{\prime}}-x_{k+1}^{r^{\prime}} x_{1}^{r}=0$, i.e., $f_{\sigma}^{r} \in I(X)$.

Te following conjecture has been verified in a number of examples using Macaulay2 [12].

Conjecture 5.10. Let $X$ be the algebraic toric set associated to an even cycle $\mathcal{G}=\mathcal{C}_{2 k}$ and let $\lambda$ be the partition $\{1,3, \ldots, 2 k-1\} \sqcup\{2,4, \ldots, 2 k\}$. If $k \geq 2$, then the set of binomials

$$
\begin{gathered}
\mathcal{B}=\left\{\left\{f_{\sigma}^{r}: \sigma=A \sqcup B \text { is a partition of }\{1, \ldots, s\} \text { with }|A|=|B|, 1 \in A \text { and } 1 \leq r \leq q-2\right\}\right. \\
\left.\cup\left\{t_{i}^{q-1}-t_{s}^{q-1}: 1 \leq i \leq s-1\right\}\right\} \backslash\left\{f_{\lambda}^{r}: 2 \leq r \leq q-2\right\}
\end{gathered}
$$

is a minimal set of generators and a Gröbner basis of $I(X)$ with respect to the reverse lexicographic order.

Remark 5.11. By Theorem 5.9 and since, for each $2 \leq r \leq q-2$, there exists $g_{r} \in S$ such that $f_{\lambda}^{r}=g_{r} f_{\lambda}^{1}$, we get that $\mathcal{B}$ is a generating set for $I(X)$. 
Corollary 5.12. Let $\mathcal{G}=\mathcal{C}_{2 k}$ be an even cycle.

(a) If $f=t^{a}-t^{b}$ is an element of $\mathcal{B}$, then $\operatorname{deg}(f)$ is at most $(q-2)(k-1)+1$.

(b) Any subset of $\mathcal{B}$ that is also generating set of $I(X)$ contains an element of the form $f_{\sigma}^{q-2}$, with $\operatorname{deg}\left(f_{\sigma}^{q-2}\right)=(q-2)(k-1)+1$.

Proof. We set $M=(q-2)(k-1)+1$. (a) If $f=t_{i}^{q-1}-t_{j}^{q-1}$ for some $i, j$, then $\operatorname{deg}(f) \leq M$ because $k \geq 2$. Assume that $f$ is not of this form. Then, $f=f_{\sigma}^{r}$ for some $1 \leq r \leq q-2$, where $\sigma$ is the partition $\sigma=A \sqcup B$ and $A, B$ are the supports of $a, b$ respectively, $|A|=|B|=k$ and $1 \in A$. Let $i$ be the cardinality of the set $\left\{j \in A: f_{\sigma}^{r}(j)=r\right\}$. Then, $\operatorname{deg}(f)=i r+(k-i) \widehat{r}$. If $r=\widehat{r}$, then $r=(q-1) / 2$ and $\operatorname{deg}(f)=k(q-1) / 2 \leq M$. We may now assume $r \neq \widehat{r}$. If $i=k$, then

$$
f_{\sigma}^{r}=f_{\lambda}^{1}=t_{1} t_{3} \cdots t_{2 k-1}-t_{2} t_{4} \cdots t_{2 k}
$$

Hence, $\operatorname{deg}\left(f_{\sigma}^{r}\right)=k \leq M$. To complete the proof we may now assume that $1 \leq i \leq k-1$. In this case, we have

$$
\begin{aligned}
\operatorname{deg}\left(f_{\sigma}^{r}\right) & =i r+(k-i) \widehat{r}=i(r-\widehat{r})+k \widehat{r} \\
& \leq(k-1)(r-\widehat{r})+k \widehat{r}=(k-1) r+\widehat{r}=r(k-2)+(q-1) \\
& \leq(q-2)(k-2)+(q-1)=(k-1)(q-2)+1 .
\end{aligned}
$$

Thus, $\operatorname{deg}\left(f_{\sigma}^{r}\right) \leq M$, as required. To prove (b) consider $\mathcal{B}^{\prime} \subset \mathcal{B}$ be a generating set of $I(X)$. Let $\sigma=\{1,3, \ldots, 2 k-3,2 k\} \sqcup\{2,4, \ldots, 2(k-1), 2 k-1\}$, then

$$
f_{\sigma}^{q-2}=t_{1}^{q-2} t_{3}^{q-2} \cdots t_{2 k-3}^{q-2} t_{2 k}-t_{2}^{q-2} t_{4}^{q-2} \cdots t_{2(k-1)}^{q-2} t_{2 k-1}
$$

is in $\mathcal{B}$ and has degree $M$. We will show that $f_{\sigma}^{q-2} \in \mathcal{B}^{\prime}$. Since $\mathcal{B}^{\prime}$ is a generating set of $I(X)$, $f_{\sigma}^{q-2}$ is a linear combination, with coefficients in $S$, of binomials in $\mathcal{B}^{\prime}$. These are binomials of the form $f_{\rho}^{m}, 1 \leq m \leq q-2, \operatorname{deg}\left(f_{\rho}^{m}\right) \leq M$, and of the form $t_{i}^{q-1}-t_{2 k}^{q-1}$ for some $1 \leq i \leq 2 k-1$. It is seen that there is a binomial in $\mathcal{B}^{\prime}, f_{\rho}^{m}=t^{a^{\prime}}-t^{b^{\prime}}$, such that

$$
t_{1}^{q-2} t_{3}^{q-2} \cdots t_{2 k-3}^{q-2} t_{2 k}=t^{c} t^{a^{\prime}}
$$

for some monomial $t^{c}$. Since $\operatorname{supp}\left(a^{\prime}\right) \subset\{1,3, \ldots, 2 k-3,2 k\}$ and $t^{a^{\prime}}-t^{b^{\prime}}$ cannot be of the form $t_{1}^{q-1}-t_{2 k}^{q-1}$, because of its degree, we deduce that $\sigma=\rho$. From the equality

$$
t_{1}^{q-2} t_{3}^{q-2} \cdots t_{2 k-3}^{q-2} t_{2 k}=t^{c} t^{a^{\prime}}=t^{c}\left(t_{1}^{m} t_{3}^{m} \cdots t_{2 k-3}^{m} t_{2 k}^{\widehat{m}}\right)
$$

we conclude that $\widehat{m}=1$, that is, $m=q-2$. Thus, $f_{\sigma}^{q-2}=f_{\rho}^{m}$.

Consider the general case when $\mathcal{G}$ is any graph. Suppose that $\mathcal{G}$ contains a subgraph $\mathcal{H} \cong \mathcal{C}_{2 k}$, isomorphic to an even order cycle. Assume without loss of generality that $t_{1}, \ldots, t_{2 k}$ are the variables of $S$ corresponding to the edges of $\mathcal{H}$. Then, given $r \in\{1, \ldots, q-2\}$ and a partition $\sigma=A \sqcup B$ of $\{1, \ldots, 2 k\}$ with $|A|=|B|=k$ and $1 \in A$, the homogeneous binomial $f_{\sigma}^{r} \in K\left[t_{1}, \ldots, t_{2 k}\right] \subset S$ clearly vanishes on the algebraic toric set associated to $\mathcal{G}$. One could conjecture that together with the binomials $t_{i}^{q-1}-t_{j}^{q-1}$, for $1 \leq i, j \leq s$, the binomials obtained in this way, going through all the even cycles of $\mathcal{G}$, would form a generating set of $I(X)$. This is not true, even for bipartite graphs, as is shown by Example 5.14. This conjecture is true if we restrict to bipartite graphs the cycles of which are vertex disjoint; as we show in Theorem 5.13 .

Suppose $\mathcal{G}$ is a bipartite graph the cycles of which have disjoint vertex sets. Let $\mathcal{H}_{1}, \ldots, \mathcal{H}_{m}$ be the subgraphs of $\mathcal{G}$ isomorphic to some even order cycle, i.e., such that $\mathcal{H}_{i} \cong \mathcal{C}_{2 k_{i}}$. Let 
$t_{\epsilon_{1}^{i}}, \ldots, t_{\epsilon_{2 k_{i}}^{i}} \in S$ be the variables associated to the edges, $e_{1}^{i}, \ldots, e_{2 k_{i}}^{i}$ of $\mathcal{H}_{i}$. Accordingly, set

$$
S_{i}=K\left[t_{\epsilon_{1}^{i}}, \ldots, t_{\epsilon_{2 k_{i}}^{i}}\right] \subset S .
$$

Finally, denote by $I_{i}(X)$ the intersection $I(X) \cap S_{i}$. Then, $I_{i}(X) \subset S_{i}$ is equal to $I\left(X_{i}\right)$, the vanishing ideal of the algebraic toric set $X_{i}$ associated to $\mathcal{H}_{i}$.

Theorem 5.13. Let $\mathcal{G}$ be a connected bipartite graph, whose (even) cycles $\mathcal{H}_{1}, \ldots, \mathcal{H}_{m}$ have disjoint vertex sets. Let $X$ be the algebraic toric set associated to $\mathcal{G}$. Then $I(X)$ is generated by the union of the set $\left\{t_{i}^{q-1}-t_{j}^{q-1}: 1 \leq i, j \leq s\right\}$ with the set $I_{1}(X) \cup \cdots \cup I_{m}(X)$.

Proof. By Theorem 4.5, it suffices to show that if $f=t^{a}-t^{b} \in I(X)$, with $a=\left(a_{1}, \ldots, a_{s}\right) \in \mathbb{N}^{s}$, $b=\left(b_{1}, \ldots, b_{s}\right) \in \mathbb{N}^{s}$, such that $\operatorname{supp}(a) \cap \operatorname{supp}(b)=\emptyset$ and $1 \leq a_{i}, b_{j} \leq q-2$, then $f$ belongs to the ideal generated by

$$
\mathcal{J}=\left\{t_{i}^{q-1}-t_{j}^{q-1}: 1 \leq i, j \leq s\right\} \cup I_{1}(X) \cup \cdots \cup I_{m}(X) .
$$

Recall that $f$ is homogeneous by Lemma 4.3. By Proposition 5.1, we know that $\operatorname{supp}(a) \cup \operatorname{supp}(b)$ is contained in the union of the sets of indices of the variables corresponding to edges of the cycles of $\mathcal{G}$. In other words, if $e_{i}$ is an edge not in any edge set of $\mathcal{H}_{1}, \ldots, \mathcal{H}_{m}$ then $i \notin \operatorname{supp}(a) \cup \operatorname{supp}(b)$. As above, denote by $t_{\epsilon_{1}^{i}}, \ldots, t_{\epsilon_{2 k_{i}}^{i}}$ the variables associated to $\mathcal{H}_{i}$. We proceed by induction on

$$
\mu_{f}=\left\{i \in\{1, \ldots, m\}:(\operatorname{supp}(a) \cup \operatorname{supp}(b)) \cap\left\{\epsilon_{1}^{i}, \ldots, \epsilon_{2 k_{i}}^{i}\right\} \neq \emptyset\right\} .
$$

Let $i \in\{1, \ldots, m\}$ be such that $(\operatorname{supp}(a) \cup \operatorname{supp}(b)) \cap\left\{\epsilon_{1}^{i}, \ldots, \epsilon_{2 k_{i}}^{i}\right\} \neq \emptyset$. Consider $a^{\sharp}, a^{b}, b^{\sharp}, b^{b} \in \mathbb{N}^{s}$ such that $\operatorname{supp}\left(a^{\sharp}\right) \cup \operatorname{supp}\left(b^{\sharp}\right) \subset\left\{\epsilon_{1}^{i}, \ldots, \epsilon_{2 k_{i}}^{i}\right\},\left(\operatorname{supp}\left(a^{b}\right) \cup \operatorname{supp}\left(b^{b}\right)\right) \cap\left\{\epsilon_{1}^{i}, \ldots, \epsilon_{2 k_{i}}^{i}\right\}=\emptyset$,

$$
t^{a}=t^{a^{\sharp}} t^{a^{b}} \quad \text { and } \quad t^{b}=t^{b^{\sharp}} t^{b^{b}} \text {. }
$$

By Corollary 5.3, $\operatorname{supp}\left(a^{\sharp}\right) \cup \operatorname{supp}\left(b^{\sharp}\right)=\left\{\epsilon_{1}^{i}, \ldots, \epsilon_{2 k_{i}}^{i}\right\}$. Since we are assuming $\mathcal{H}_{1}, \ldots, \mathcal{H}_{m}$ have disjoint vertex sets, setting $t_{\ell}=1$ for all $\ell \notin\left\{\epsilon_{1}^{i}, \ldots, \epsilon_{2 k_{i}}^{i}\right\}$ is equivalent to setting in $\mathbf{x} \in\left(K^{*}\right)^{n}$, $x_{\ell}=1$ for all $\ell \notin V_{\mathcal{H}_{i}}$. Hence, making these substitutions and running the argument of the proof of Theorem 5.13, we see that $t^{a^{\sharp}}-t^{b^{\sharp}}=f_{\sigma}^{r}$, where $r=\left(a^{\sharp}\right)_{\epsilon_{1}^{i}} \in\{1, \ldots, q-2\}$, (assuming that $\left.\epsilon_{1}^{i} \in \operatorname{supp}\left(a^{\sharp}\right)\right)$, and where $\sigma$ is the $\operatorname{partition} \operatorname{supp}\left(a^{\sharp}\right) \sqcup \operatorname{supp}\left(b^{\sharp}\right)=\left\{\epsilon_{1}^{i}, \ldots, \epsilon_{2 k_{i}}^{i}\right\}$.

Suppose that $\mu_{f}=1$. Then $a^{b}=b^{b}=0 \in \mathbb{N}^{s}, f_{\sigma}^{r}$ is homogeneous and we are done.

Suppose that every binomial $g=t^{a}-t^{b} \in I(X)$ with $\mu_{g} \leq m^{\prime}<m$ is in the ideal generated by $\mathcal{J}$. Let $f=t^{a}-t^{b} \in I(X)$ be a binomial with $\mu_{f}=m^{\prime}+1$. Let $i \in\{1, \ldots, m\}$ be such that $(\operatorname{supp}(a) \cup \operatorname{supp}(b)) \cap\left\{\epsilon_{1}^{i}, \ldots, \epsilon_{2 k_{i}}^{i}\right\} \neq \emptyset$. Consider, as above, $a^{\sharp}, a^{b}, b^{\sharp}, b^{b} \in \mathbb{N}^{s}$ such that $t^{a}=t^{a^{\sharp}} t^{a^{b}}$ and $t^{b}=t^{b^{\sharp}} t^{b^{b}}$. Repeating the previous argument we deduce that $t^{a^{\sharp}}-t^{b^{\sharp}}=f_{\sigma}^{r}$ where, $r=\left(a^{\sharp}\right)_{\epsilon_{1}^{i}}$ and $\sigma=\operatorname{supp}\left(a^{\sharp}\right) \sqcup \operatorname{supp}\left(b^{\sharp}\right)$. However, notice that in this case $f_{\sigma}^{r}$ is not necessarily homogeneous. Assume that $\left|\operatorname{supp}\left(a^{\sharp}\right)\right| \geq\left|\operatorname{supp}\left(b^{\sharp}\right)\right|$. Let $\delta \in \mathbb{N}^{s}$ be such that $\epsilon_{1}^{i} \notin \operatorname{supp}(\delta) \subset \operatorname{supp}\left(a^{\sharp}\right), \delta_{\ell}=a_{\ell}^{\sharp}$ for all $\ell \in \operatorname{supp}(\delta)$ and $\left|\operatorname{supp}\left(a^{\sharp}-\delta\right)\right|=k_{i}\left(\right.$ where $2 k_{i}$ is the order of $\left.\mathcal{H}_{i}\right)$. Set $h=|\operatorname{supp}(\delta)|$, $a^{\prime}=a^{\sharp}-\delta$ and let $b^{\prime} \in \mathbb{N}^{s}$ be obtained by applying $h$ times Lemma 5.7 to $\sigma=\operatorname{supp}\left(a^{\sharp}\right) \sqcup \operatorname{supp}\left(b^{\sharp}\right)$. Then $b^{\prime}=b^{\sharp}+\hat{\delta}$, where $\hat{\delta}$ has the same support as $\delta$ and $(\hat{\delta})_{\ell}=q-1-\delta_{\ell}$, for every $\ell \in \operatorname{supp}(\hat{\delta})$. Set $\sigma^{\prime}=\operatorname{supp}\left(a^{\prime}\right) \sqcup \operatorname{supp}\left(b^{\prime}\right)$. Then $f_{\sigma^{\prime}}^{r}=t^{a^{\prime}}-t^{b^{\prime}}$ is homogeneous and belongs to $I_{i}(X)$. Moreover,

$$
\begin{gathered}
f=t^{a}-t^{b}=t^{a^{\prime}} t^{\delta} t^{a^{b}}-t^{b^{\sharp}} t^{b^{b}}=t^{a^{\prime}} t^{\delta} t^{a^{b}}-t^{b^{\prime}} t^{\delta} t^{a^{b}}+t^{b^{\prime}} t^{\delta} t^{a^{b}}-t^{b^{\sharp}} t^{b^{b}} \\
=f_{\sigma^{\prime}}^{r} t^{\delta} t^{a^{b}}+t^{b^{\sharp}}\left(t^{\widehat{\delta}} t^{\delta} t^{a^{b}}-t^{b^{b}}\right) .
\end{gathered}
$$


Now $(\hat{\delta})_{\ell}+\delta_{\ell}=q-1$, for all $\ell \in \operatorname{supp}(\delta)$ and since $f$ is homogeneous, $h=|\operatorname{supp}(\delta)|>$ $\left|\operatorname{supp}\left(b^{b}\right)\right|$. Choose $\ell_{1}, \ldots, \ell_{h} \in \operatorname{supp}\left(b^{b}\right), h$ distinct indices. Let $\gamma \in \mathbb{N}^{s}$ to be such that $\operatorname{supp}(\gamma)=$ $\left\{\ell_{1}, \ldots, \ell_{h}\right\}$ and $(\gamma)_{\ell_{j}}=q-1$, for $j=1, \ldots, h$. Then $t^{\delta} t^{\widehat{\delta}}-t^{\gamma}$ is in the ideal of $S$ generated by $\mathcal{J}$, since it is in the ideal of the torus. We have

$$
f=f_{\sigma^{\prime}}^{r} t^{\delta} t^{a^{b}}+t^{b^{\sharp}}\left(t^{\delta} t^{\widehat{\delta}} t^{a^{b}}-t^{b^{b}}\right)=f_{\sigma^{\prime}}^{r} t^{\delta} t^{a^{b}}+t^{b^{\sharp}} t^{a^{b}}\left(t^{\delta} t^{\widehat{\delta}}-t^{\gamma}\right)+t^{b^{\sharp}}\left(t^{\gamma} t^{a^{b}}-t^{b^{b}}\right) .
$$

Let $\gamma^{\sharp} \in \mathbb{N}^{s}$ be such that $\operatorname{supp}\left(\gamma^{\sharp}\right)=\left\{\ell_{1}, \ldots, \ell_{h}\right\}$ and $\left(\gamma^{\sharp}\right)_{\ell_{j}}=\left(b^{b}\right)_{\ell_{j}}$, for $j=1, \ldots, h$ and set $\gamma^{b}=\gamma-\gamma^{\sharp}$ and $b^{\natural}=b^{b}-\gamma^{\sharp}$. Then,

$$
f=f_{\sigma^{\prime}}^{r} t^{\delta} t^{a^{b}}+t^{b^{\sharp}} t^{a^{b}}\left(t^{\delta^{*}}-t^{\gamma}\right)+t^{b^{\sharp}} t^{\gamma^{\sharp}}\left(t^{\gamma^{b}} t^{a^{b}}-t^{b^{\natural}}\right),
$$

where $g=t^{\gamma^{b}} t^{a^{b}}-t^{b^{\natural}}$ is a homogeneous binomial with $\mu_{g} \leq m^{\prime}$. Hence, by induction, $g$, and therefore $f$, are in the ideal generated by $\mathcal{J}$.

In Example 5.14, we show that Theorem 5.13 does not hold for general connected bipartite graphs.
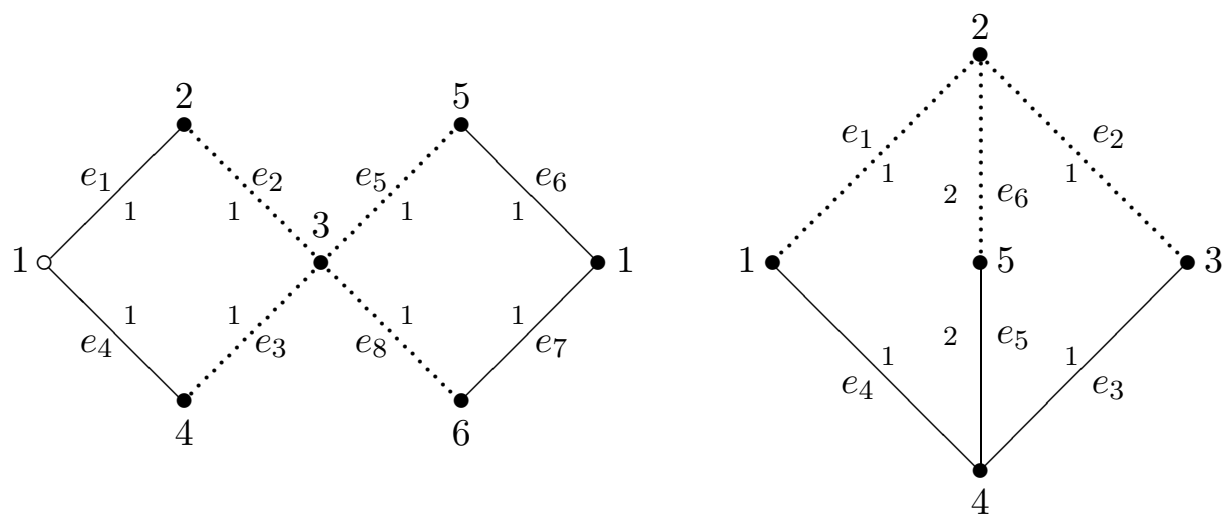

FiguRE 4.

Example 5.14. Let $\mathcal{G}_{1}$ and $\mathcal{G}_{2}$ be the two graphs in Figure 4 (from left to right) and assume that $q=5$. Notice that we are identifying the two vertices, labeled by 1 , in the representation of $\mathcal{G}_{1}$. Thus, $\mathcal{G}_{1}$ is a bipartite graph with six vertices and eight edges. Denote by $X_{1}$ and $X_{2}$, respectively, the corresponding algebraic toric sets. Then, using Macaulay2 [12, we found that the binomial $t_{1} t_{4} t_{6} t_{7}-t_{2} t_{3} t_{5} t_{8}$ is in a minimal generating set of $I\left(X_{1}\right)$. In this case, the argument of the proof of Theorem 5.13 does not work, to the extent that if we set $t_{1}, t_{2}, t_{3}, t_{4}$ equal to 1 , the resulting binomial, $t_{6} t_{7}-t_{5} t_{8}$, albeit homogeneous, is not of the type $f_{\sigma}^{r}$ for any partition $\sigma$ of $\{5,6,7,8\}$. The same can be said for the binomial resulting from substituting to 1 the variables $t_{5}, t_{6}, t_{7}, t_{8}$. As to the vanishing ideal of $X_{2}$, we found that there exists a minimal generating set containing $t_{1} t_{2} t_{5}^{2}-t_{3} t_{4} t_{5}^{2}$, which, when restricted to any of the 3 cycles in $\mathcal{G}_{2}$ is not of the type $f_{\sigma}^{r}$ for any partition of the corresponding index set.

\section{The Regularity of $R / I(X)$}

In this section we address the question of computing the regularity of $S / I(X)$ for an algebraic toric set $X$ parameterized by a bipartite graph. Theorem 6.3 gives an upper bound for the regularity of $S / I(X)$ for a general bipartite graph. If $X$ is the algebraic toric set parameterized by an even cycle of length $2 k$, by Proposition 2.4 and Corollary 5.12 , we get

$$
\operatorname{bigdeg} I(X)-1=(q-2)(k-1) \leq \operatorname{reg} S / I(X) .
$$


This inequality is already known in the literature, see [8, Corollary 3.1] and [24, Corollary 2.19]. We will show that the regularity of $S / I(X)$ is in fact equal to $(q-2)(k-1)$, and generalize this result by giving a formula for the regularity of any connected bipartite graph whose cycles have disjoint vertex sets. In the proof of Theorem 6.2. we show the inequality above as an easy consequence of the description of the generators of the ideal $I(X)$.

Lemma 6.1. Let $1 \leq i \leq s-2$. Consider the $K$-automorphism $\sigma_{i}: S \rightarrow S$ defined by exchanging $t_{i}$ with $t_{i+2}$ and leaving all other variables fixed. Then, $\sigma_{i}$ permutes the elements of the set of all $f_{\sigma}^{r} \in I(X)$, for $r \in\{1, \ldots, q-2\}$ and $\sigma=A \sqcup B$ a partition of $\{1, \ldots, s\}$ with $|A|=|B|$.

Proof. Let $f_{\sigma}^{r}$ be a binomial associated to $r \in\{1, \ldots, q-2\}$ and $\sigma=A \sqcup B$ a partition of $\{1, \ldots, s\}$. Thus, $f_{\sigma}^{r}=t^{a}-t^{b}$ where $A=\operatorname{supp}(a), B=\operatorname{supp}(b), a_{\ell}=\rho_{\sigma}^{r}(\ell)$, for all $\ell \in \operatorname{supp}(a)$ and $b_{\ell}=\rho_{\sigma}^{r}(\ell)$, for all $\ell \in \operatorname{supp}(b)$. As $\rho_{\sigma}^{r}(\ell)=\rho_{\sigma}^{r}(\ell+2)$ if and only if $\ell$ and $\ell+2$ are in the same part of the partition, if $i$ and $i+2$ are in the same part of the partition then $\sigma_{i}\left(f_{\sigma}^{r}\right)=f_{\sigma}^{r}$. Suppose that $i$ and $i+2$ are in different parts of the partition and therefore that $\rho_{\sigma}^{r}(i+2)=\widehat{\rho_{\sigma}^{r}(i)}$. Without loss in generality we may write $f_{\sigma}^{r}=t_{i}^{a_{i}} t^{a^{\prime}}-t_{i+1}^{\widehat{a_{i}}} t^{b^{\prime}}$, where $\operatorname{supp}\left(a^{\prime}\right)=\operatorname{supp}(a) \cup\{i\}$ and $\operatorname{supp}\left(b^{\prime}\right)=\operatorname{supp}(b) \cup\{i+2\}$. In this situation, we apply Lemma 5.7 twice, transferring $i$ to the part it does not belong to, and proceeding similarly with $i+2$. Let $\sigma^{\prime}$ be the partition of $\{1, \ldots, s\}$ obtained in this way and consider the resulting binomial $f_{\sigma^{\prime}}^{r}$. By Lemma 5.7 we see that $f_{\sigma^{\prime}}^{r}=t_{i+2}^{a_{i}} t^{a^{\prime}}-t_{i}^{\widehat{a_{i}}} t^{b^{\prime}}=\sigma_{i}\left(f_{\sigma}^{r}\right)$.

Theorem 6.2. Let $X$ be the algebraic toric set associated to an even order cycle $\mathcal{G}=\mathcal{C}_{2 k}$. Then $\operatorname{reg} S / I(X)=(q-2)(k-1)$.

Proof. Recall that $k \geq 2$. Denote by $R$ the graded ring $S / I(X)$. Consider $t_{1} \in S$. Since $t_{1}$ is regular on $R$, we have the following exact sequence of graded $S$-modules:

$$
0 \longrightarrow R[-1] \stackrel{t_{1}}{\longrightarrow} R \longrightarrow R /\left(t_{1}\right) \longrightarrow 0,
$$

where $R[-1]$ is the graded $S$-module obtained by a shift in the graduation, i.e., $R[-1]_{i}=R_{i-1}$. Recall that $H_{X}(d)$ is, by definition, $\operatorname{dim}_{K}(S / I(X))_{d}$, and since $S / I(X)$ is a 1-dimensional ring, the regularity of $S / I(X)$ is the least integer $l$ for which $H_{X}(d)$ is equal to some constant (indeed equal to $|X|)$ for all $d \geq l$. Now, from (6.1) we get $H_{X}(d)-H_{X}(d-1)=\operatorname{dim}_{K}\left(R /\left(t_{1}\right)\right)_{d}$. Hence $\operatorname{reg} S / I(X)=\operatorname{reg} R /\left(t_{1}\right)-1$. For $d \geq 0$, we define

$$
h_{d}:=\operatorname{dim}_{K}\left(R /\left(t_{1}\right)\right)_{d}=H_{X}(d)-H_{X}(d-1) .
$$

We start by showing that $\operatorname{reg} S / I(X) \leq(q-2)(k-1)$. If we show that $h_{d}=0$, for $d \geq$ $(q-2)(k-1)+1$, then $H_{X}(d-1)=H_{X}(d)$, for $d-1 \geq(q-2)(k-1)$, and our result follows. Set $S^{\prime}=K\left[t_{2}, \ldots, t_{s}\right]$. There is a surjection of graded $S^{\prime}$-modules

$$
\varphi: S^{\prime} \longrightarrow S /\left(I(X), t_{1}\right) \cong R /\left(t_{1}\right)
$$

defined by $\varphi(f)=f+\left(I(X), t_{1}\right)$, for every $f \in S^{\prime}$. Set $I^{\prime}(X)=\operatorname{Ker}(\varphi)$, so that

$$
S^{\prime} / I^{\prime}(X) \cong S /\left(I(X), t_{1}\right) .
$$

Then, $I^{\prime}(X)$ is a monomial ideal generated by the monomials obtained by setting $t_{1}=0$ in the generators of $I(X)$; in particular it is generated by $t_{j}^{q-1}$, for $2 \leq j \leq s$ and by the monomials $t^{b}$ in some $f_{\sigma}^{r}=t^{a}-t^{b}$, for $r \in\{1, \ldots, q-2\}$ and $\sigma$ a partition of $\{1, \ldots, s\}$ into 2 parts of equal cardinality. To show that $h_{d}=0$, for $d \geq(q-2)(k-1)+1$, it is enough to show that every monomial $M$ in $S^{\prime}$ of degree $\geq(q-2)(k-1)+1$ belongs to $I^{\prime}(X)$. Since $t_{j}^{q-1} \in I^{\prime}(X)$ for all 
$2 \leq j \leq s$, we may assume that there is no $j$ for which $t_{j}^{q-1}$ divides the monomial $M$ in question. Let us write it in the following way:

$$
M=t_{2}^{b_{1}} t_{4}^{b_{2}} \cdots t_{2 k}^{b_{k}} t_{3}^{c_{1}} t_{5}^{c_{2}} \cdots t_{2 k-1}^{c_{k-1}},
$$

with $0 \leq b_{i}, c_{j} \leq q-2$. We want to show that there exists $f_{\sigma}^{r}=t^{a}-t^{b} \in I(X)$ such that $t^{b}$ divides $M$. By Lemma 6.1, if $t^{b}$ divides $M$ and there exists $r, \sigma$ such that $f_{\sigma}^{r}=t^{a}-t^{b}$, then, for all $i \in\{2, \ldots, s-2\}, \sigma_{i}\left(t^{b}\right)$ divides $\sigma_{i}(M)$ and there exists $\sigma^{\prime}$ such that $f_{\sigma^{\prime}}^{r}=t^{a^{\prime}}-\sigma_{i}\left(t^{b}\right)$. Hence, we may assume that $c_{1} \leq c_{2} \leq \cdots \leq c_{k-1}$ and that $b_{1} \geq b_{2} \geq \cdots \geq b_{k}$. There are two cases. If $b_{k}>0$, then $M$ is divisible by $t_{2} t_{4} \cdots t_{2 k}$, which belongs to $I^{\prime}(X)$, since for $\sigma=\{1,3, \ldots, 2 k-1\} \sqcup\{2,4, \ldots, 2 k\}$, we have $f_{\sigma}^{1}=t_{1} t_{3} \cdots t_{2 k-1}-t_{2} t_{4} \cdots t_{2 k}$. The second case is for $b_{k}=0$. In this case, from

$$
\operatorname{deg} M=\sum_{i=1}^{k-1}\left(b_{i}+c_{i}\right) \geq(q-2)(k-1)+1
$$

we deduce that there exists $j \in\{1, \ldots, k-1\}$ such that $b_{j}+c_{j} \geq q-1$. Since $c_{j} \leq q-2$ we get $b_{j} \geq 1$. Set $r=b_{j}$. Notice that then $c_{j} \geq q-1-b_{j}=q-1-r=\widehat{r}$. Consider the set given by $B=\{2,4, \ldots, 2 j, 2 j+1,2 j+3, \ldots, 2 k-1\}$ and let $\sigma=A \sqcup B$ be the partition of $\{1, \ldots, s\}$ that it determines. Then:

$$
f_{\sigma}^{r}=\left(t_{1} t_{3} \cdots t_{2 j-1}\right)^{r}\left(t_{2 j+2} \cdots t_{2 k-2} t_{2 k}\right)^{\widehat{r}}-\left(t_{2} t_{4} \cdots t_{2 j}\right)^{r}\left(t_{2 j+1} t_{2 j+3} \cdots t_{2 k-1}\right)^{\widehat{r}} \in I(X) .
$$

Accordingly, $\left(t_{2} t_{4} \cdots t_{2 j}\right)^{r}\left(t_{2 j+1} t_{2 j+3} \cdots t_{2 k-1}\right)^{\widehat{r}} \in I^{\prime}(X)$. Since $b_{l} \geq b_{j}=r$, for all $1 \leq l \leq j$, we deduce that $t_{2 l}^{r}$ divides $M$, for all $1 \leq l \leq j$. Since $\widehat{r} \leq c_{j} \leq c_{l}$, for all $j \leq l \leq k-1$, we deduce that $t_{2 l+1}^{\widehat{r}}$ divides $M$, for all $j \leq l \leq k-1$. In conclusion, $\left(t_{2} t_{4} \cdots t_{2 j}\right)^{r}\left(t_{2 j+1} t_{2 j+3} \cdots t_{2 k-1}\right)^{\widehat{r}}$ divides $M$ and hence $M \in I^{\prime}(X)$.

Let us now show that $\operatorname{reg} S / I(X) \geq(q-2)(k-1)$. If we show that $h_{d} \neq 0$, i.e., $h_{d}>0$, for $d=(q-2)(k-1)$, then $H_{X}(d-1)<H_{X}(d)$, for $d=(q-2)(k-1)$, and our result follows. It suffices to produce a monomial $M$ of degree $d=(q-2)(k-1)$, such that $M \in\left(S^{\prime}\right)_{d}$ but $M \notin\left(I^{\prime}(X)\right)_{d}$. Consider

$$
M=\left(t_{2} \cdots t_{k}\right)^{q-2} \in\left(S^{\prime}\right)_{d} .
$$

Suppose $M \in I^{\prime}(X)$. Then, as we have seen above,

$$
M=\sum_{j=2}^{s} g_{j} t_{j}^{q-1}+\sum_{\sigma, r} h_{\sigma, r} t^{b}
$$

where $g_{j}, h_{\sigma, r} \in S^{\prime}, f_{\sigma}^{r}=t^{a}-t^{b}$ and the second summation runs over all partitions $\sigma$ of $\{1, \ldots, s\}$ into 2 parts of equal cardinality and $r \in\{1, \ldots, q-2\}$. Since $M$ is a monomial and its degree in each one of the variables is $q-2$, we deduce that $M$ must be a monomial of the form

$$
M=h_{\sigma, r} t^{b}
$$

for $h_{\sigma, r} \in S^{\prime}$, one partition $\sigma$ of $\{1, \ldots, s\}$ into 2 parts of equal cardinality, one $r \in\{1, \ldots, q-2\}$ and $f_{\sigma}^{r}=t^{a}-t^{b}$. But this is not possible because the monomial $M$ has $k-1$ variables, while $h_{\sigma, r} t^{b}$ has at least $k$ variables. We conclude that $M \notin I^{\prime}(X)$.

Theorem 6.3. Let $\mathcal{G}$ be a bipartite graph. Let $\mathcal{H}_{1}, \ldots, \mathcal{H}_{m}$ be subgraphs of $\mathcal{G}$ isomorphic to (even) cycles $\mathcal{H}_{i} \cong \mathcal{C}_{2 k_{i}}$ that have disjoint edge sets. Then

$$
\operatorname{reg} S / I(X) \leq(q-2)\left(s-\sum_{i=1}^{m} k_{i}-1\right) .
$$


Proof. For all $1 \leq i \leq m$, let $t_{\epsilon_{1}^{i}}, \ldots, t_{\epsilon_{2 k_{i}}^{i}}$ be the variables associated to the edges of $\mathcal{H}_{i}$.

Without loss of generality, assume that $t_{1}=t_{\epsilon_{1}^{1}}, t_{2}=t_{\epsilon_{1}^{2}}, \ldots, t_{m}=t_{\epsilon_{1}^{m}}$.

Denote by $R$ the quotient $S / I(X)$ and, for $1 \leq i \leq m$, let

$$
R_{i}=R /\left(t_{1}, \ldots, t_{i}\right) .
$$

Since $t_{1}$ is a regular element of $R$, we have the following short exact sequence of graded $S$ modules:

$$
0 \longrightarrow R[-1] \stackrel{t_{1}}{\longrightarrow} R \longrightarrow R_{1} \longrightarrow 0 .
$$

Furthermore, for all $1 \leq i \leq m-1$, we have exact sequences of graded $S$-modules:

$$
R_{i}[-1] \stackrel{t_{i+1}}{\longrightarrow} R_{i} \longrightarrow R_{i+1} \longrightarrow 0 .
$$

Claim 1. For all $1 \leq i \leq m, t_{j}^{q-1}=0$ in $R_{i}$, for all $1 \leq j \leq s$.

Proof of Claim 1. Since $t_{j}^{q-1}-t_{i}^{q-1} \in I(X)$ and $t_{i}^{q-1}=0$ in $R_{i}$, we deduce that $t_{j}^{q-1}=0$ in $R_{i}$, for all $1 \leq j \leq s$.

Claim 2. If there exists a nonnegative integer $\ell$ such that $\left(R_{i+1}\right)_{d}=0$, for all $d \geq \ell$, then $\left(R_{i}\right)_{d}=0$ for all $d \geq \ell+q-2$, where $1 \leq i \leq m-1$.

Proof of Claim 2. If $\left(R_{i+1}\right)_{d}=0$, for $d \geq \ell$ then from (6.3) we deduce that for all $d \geq \ell$ the maps $\left(R_{i}\right)_{d-1} \stackrel{t_{i+1}}{\longrightarrow}\left(R_{i}\right)_{d}$ are surjective, i.e., $\left(R_{i}\right)_{d}=t_{i+1}\left(R_{i}\right)_{d-1}$, for all $d \geq \ell$. Iterating and using Claim 1, we get: $\left(R_{i}\right)_{d+q-2}=t_{i+1}^{q-1}\left(R_{i}\right)_{d-1}=0$, i.e., $\left(R_{i}\right)_{d}=0$ for all $d \geq \ell+q-2$.

Claim 3. Let $t^{a}$ be a monomial in $S$. Suppose that the degree of $t^{a}$ in the variables associated to $\mathcal{H}_{i}$ is $\geq(q-2)\left(k_{i}-1\right)+1$. Then $t^{a}=0$ in $R_{i}$.

Proof of Claim 3. We may assume that $t_{i}$ does not divide $t^{a}$. Defining

$$
S_{i}:=K\left[t_{\epsilon_{1}^{i}}, \ldots, t_{\epsilon_{2 k_{i}}^{i}}\right] \text {, }
$$

we have $I\left(X_{i}\right) \subset S_{i}$, where $X_{i} \subseteq \mathbb{P}^{2 k_{i}-1}$ is the set of points parameterized by the edges of the cycle $\mathcal{H}_{i}$. It is straightforward to check that $I\left(X_{i}\right) \subset I(X) \subset S$. Let $t^{a}=t^{b} t^{c}$, where $t^{b}$ is a monomial in $t_{\epsilon_{1}^{i}}, \ldots, t_{\epsilon_{2 k_{i}}^{i}}$. It suffices to show $t^{b}=0$ in $S_{i} /\left(I\left(X_{i}\right)+t_{i}\right)$, but since $t^{b}$ has degree $\geq(q-2)\left(k_{i}-1\right)+1$, we can run the same argument as in the proof of Theorem 6.2.

Claim 4. Let $\ell_{0}=(q-2)\left(\sum_{i=1}^{m}\left(k_{i}-1\right)\right)+(q-2)\left(s-\sum_{i=1}^{m} 2 k_{i}\right)+1$. Then $\left(R_{m}\right)_{d}=0, \forall d \geq \ell_{0}$.

Proof of Claim 4. Let $t^{a}$ be a monomial of degree $d \geq \ell_{0}$. In view of Claim 3, we may assume that the degree of $t^{a}$ in the variables associated to $\mathcal{H}_{i}$ is $\leq(q-2)\left(k_{i}-1\right)$. Then, the degree of $t^{a}$ in the remaining $s-\sum_{i=1}^{m} 2 k_{i}$ variables is $\geq(q-2)\left(s-\sum_{i=0}^{m} 2 k_{i}\right)+1$ which implies that one of them is raised to a power $\geq q-1$ and therefore, by Claim $1, t^{a}=0$ in $R_{m}$.

We now finish the proof of the theorem. Notice that $\ell_{0}=(q-2)\left(s-\sum_{i=1}^{m}\left(k_{i}+1\right)\right)+1$. Combining Claim 2 with Claim 4 we deduce that $\left(R_{1}\right)_{d}=0$, for all $d \geq \ell_{0}+(m-1)(q-2)$. Now $\ell_{0}+(m-1)(q-2)=(q-2)\left(s-\sum_{i=1}^{m} k_{i}-1\right)+1$ and using (6.2) we see that $(R)_{d-1} \stackrel{t_{1}}{\longrightarrow}$ $(R)_{d}$ is an isomorphism for all $d \geq(q-2)\left(s-\sum_{i=1}^{m} k_{i}-1\right)+1$. This means that the Hilbert function of $R$ satisfies: $H_{X}(d-1)=H_{X}(d)$, for $d-1 \geq(q-2)\left(s-\sum_{i=1}^{m} k_{i}-1\right)$. Hence, $\operatorname{reg} R \leq(q-2)\left(s-\sum_{i=1}^{m} k_{i}-1\right)$. 
Remark 6.4. Notice we do not assume that $\mathcal{G}$ is connected nor do we assume that any 2 cycles, $\mathcal{H}_{1}$ and $\mathcal{H}_{2}$, in $\mathcal{G}$ have disjoint edge or vertex sets. In fact, we can apply the bound of Theorem 6.3 to both graphs in Figure 4. For $\mathcal{G}_{1}$, on the left, we should use both cycles of order 4. We obtain reg $S / I\left(X_{1}\right) \leq 3(8-4-1)=9$. Using Macaulay2 [12], for $q=5$, we checked that this is the actual value of the regularity. For $\mathcal{G}_{2}$, on the right, we may only use one of the cycles. Then, Theorem 6.3 yields reg $S / I\left(X_{2}\right) \leq(q-2)(6-2-1)=3(q-2)$, which, for $q=5$, is not sharp, as the value of reg $S / I\left(X_{2}\right)$ is 6 . The inequality of Theorem 6.3 is an improvement of the inequality given in [24, Corollary 2.31].

Corollary 6.5. Let $\mathcal{G}$ be a connected bipartite graph, the (even) cycles of which, $\mathcal{H}_{1}, \ldots, \mathcal{H}_{m}$, with $\mathcal{H}_{i} \cong \mathcal{C}_{2 k_{i}}$, have disjoint vertex sets. Then

$$
\operatorname{reg} S / I(X)=(q-2)\left(s-\sum_{i=1}^{m} k_{i}-1\right) .
$$

Proof. Let $t_{\epsilon_{1}^{i}}, \ldots, t_{\epsilon_{2 k_{i}}^{i}} \in S$ be the set of variables associated to the edges, $e_{1}^{i}, \ldots, e_{2 k_{i}}^{i}$ of the even cycle $\mathcal{H}_{i}$. We set

$$
S_{i}=K\left[t_{\epsilon_{1}^{i}}, \ldots, t_{\epsilon_{2 k_{i}}^{i}}\right] \subset S,
$$

and denote by $I_{i}(X)$ the intersection $I(X) \cap S_{i}$. Then, $I_{i}(X) \subset S_{i}$ is the vanishing ideal of the algebraic toric set associated to $\mathcal{H}_{i}$. By Theorem 5.13, $I(X)$ is generated by the set

$$
\mathcal{J}=\left\{t_{i}^{q-1}-t_{j}^{q-1}: 1 \leq i, j \leq s\right\} \cup I_{1}(X) \cup \cdots \cup I_{m}(X) .
$$

We proceed by induction on the number of edges of $\mathcal{G}$. If $\mathcal{G}$ is an even cycle, the result follows from Theorem 6.2. We may assume that $e_{s}$ is an edge of $\mathcal{G}$ that does not lie on any cycle of $\mathcal{G}$ and that $t_{s}$ is the variable that corresponds to $e_{s}$. For simplicity of notation, we identify the edge $e_{i}$ with the variable $t_{i}$ for $i=1, \ldots, s$ and refer to $t_{i}$ as an edge of the graph $\mathcal{G}$. Consider the graph $\mathcal{G}_{1}$ whose edge set is $\left\{e_{1}, \ldots, e_{s-1}\right\}$ (the edge set of $\mathcal{G}$ minus the edge $e_{s}$ ), and whose vertex set is the set of endpoints of the edges $e_{1}, \ldots, e_{s-1}$. Let $X_{1}$ be the algebraic toric set parameterized by the edges of $\mathcal{G}_{1}$. Clearly $\mathcal{G}_{1}$ is a bipartite graph whose (even) cycles are again $\mathcal{H}_{1}, \ldots, \mathcal{H}_{m}$.

Case (I): The graph $\mathcal{G}_{1}$ is connected. Let $A\left(X_{1}\right)=K\left[t_{1}, \ldots, t_{s-1}\right] / I\left(X_{1}\right)$ be the coordinate ring of $X_{1}$ and let $F_{X_{1}}(t)$ be the Hilbert series of $A\left(X_{1}\right)$. The Hilbert series can be uniquely written as $F_{X_{1}}(t)=g_{1}(t) /(1-t)$, where $g_{1}(t)$ is a polynomial of degree equal to the regularity of $A\left(X_{1}\right)$. Because $\mathcal{G}_{1}$ is a connected bipartite graph and has the same cycles as $\mathcal{G}$, by Theorem 5.13 , the vanishing ideal $I\left(X_{1}\right)$ is generated by the set

$$
\mathcal{J}_{1}=\left\{t_{i}^{q-1}-t_{j}^{q-1}: 1 \leq i, j \leq s-1\right\} \cup I_{1}(X) \cup \cdots \cup I_{m}(X)
$$

(notice that $I_{j}(X)=I_{j}\left(X_{1}\right)$, for $\left.j=1, \ldots, m\right)$. Hence, there is an exact sequence

$$
0 \rightarrow A\left(X_{1}\right)[-(q-1)] \stackrel{t_{1}^{q-1}}{\longrightarrow} A\left(X_{1}\right) \longrightarrow C=K\left[t_{1}, \ldots, t_{s-1}\right] /\left(I_{1}(X), \ldots, I_{m}(X), t_{1}^{q-1}, \ldots, t_{s-1}^{q-1}\right) \rightarrow 0 .
$$

As a consequence, we get that the Hilbert series $F(C, t)$ of $C$ is given by

$$
F(C, t)=F_{X_{1}}(t)\left(1-t^{q-1}\right)=g_{1}(t)\left(1+t+\cdots+t^{q-2}\right),
$$

and $\operatorname{deg} F(C, t)=(q-2)+\operatorname{reg} A\left(X_{1}\right)$. Since $\mathcal{G}_{1}$ is a connected bipartite graph ant its even cycles have disjoint vertex sets, by induction we get reg $A\left(X_{1}\right)=(q-2)\left(s-1-\sum_{i=1}^{m} k_{i}-1\right)$, and therefore,

$$
\operatorname{deg} F(C, t)=(q-2)\left(s-\sum_{i=1}^{m} k_{i}-1\right) .
$$


From the exact sequence

$$
0 \rightarrow(S / I(X))[-1] \stackrel{t_{s}}{\longrightarrow} S / I(X) \longrightarrow S /\left(t_{s}, I(X)\right) \rightarrow 0,
$$

we get that $F_{X}(t)=F\left(S /\left(t_{s}, I(X)\right), t\right) /(1-t)$. Thus $\operatorname{reg}(S / I(X))=\operatorname{deg} F\left(S /\left(t_{s}, I(X)\right), t\right)$. Using the isomorphism

$$
S /\left(t_{s}, I(X)\right) \simeq K\left[t_{1}, \ldots, t_{s-1}\right] /\left(t_{1}^{q-1}, \ldots, t_{s-1}^{q-1}, I_{1}(X), \ldots, I_{m}(X)\right),
$$

we obtain that $C \simeq S /\left(t_{s}, I(X)\right)$. Hence, by Eq. (6.4), the desired formula follows.

Case (II): The graph $\mathcal{G}_{1}$ is disconnected. It is not hard to show that $\mathcal{G}_{1}$ has exactly two connected components $\mathcal{G}_{1}^{\prime}, \mathcal{G}_{1}^{\prime \prime}$. Let $E_{1}^{\prime}, E_{1}^{\prime \prime}$ be the edge sets of $\mathcal{G}_{1}^{\prime}, \mathcal{G}_{1}^{\prime \prime}$ respectively and let $X_{1}^{\prime}, X_{1}^{\prime \prime}$ be the algebraic toric sets parameterized by the edges of $\mathcal{G}_{1}^{\prime}, \mathcal{G}_{1}^{\prime \prime}$ respectively. We may assume that $\mathcal{H}_{1}, \ldots, \mathcal{H}_{r}$ are the cycles of $\mathcal{G}_{1}^{\prime}$ and $\mathcal{H}_{r+1}, \ldots, \mathcal{H}_{m}$ are the cycles of $\mathcal{G}_{1}^{\prime \prime}$. By Theorem [5.13, we have that $I\left(X_{1}^{\prime}\right)$ and $I\left(X_{1}^{\prime \prime}\right)$ are generated by

$$
\begin{aligned}
& \mathcal{J}_{1}^{\prime}=\left\{t_{i}^{q-1}-t_{j}^{q-1}:\right.\left.t_{i}, t_{j} \in E_{1}^{\prime}\right\} \cup I_{1}(X) \cup \cdots \cup I_{r}(X) \text { and } \\
& \mathcal{J}_{1}^{\prime \prime}=\left\{t_{i}^{q-1}-t_{j}^{q-1}: t_{i}, t_{j} \in E_{1}^{\prime \prime}\right\} \cup I_{r+1}(X) \cup \cdots \cup I_{m}(X),
\end{aligned}
$$

respectively. We set

$$
C_{1}^{\prime}=K\left[E_{1}^{\prime}\right] /\left(\left\{t_{i}^{q-1}\right\}_{t_{i} \in E_{1}^{\prime}}, I_{1}(X), \ldots, I_{r}(X)\right), \quad C_{1}^{\prime \prime}=K\left[E_{1}^{\prime \prime}\right] /\left(\left\{t_{i}^{q-1}\right\}_{t_{i} \in E_{1}^{\prime \prime}}, I_{r+1}(X), \ldots, I_{m}(X)\right) .
$$

By the arguments that we used to prove Case (I), and using the induction hypothesis, we get

$$
\operatorname{deg} F\left(C_{1}^{\prime}, t\right)=(q-2)\left(\left|E_{1}^{\prime}\right|-\sum_{i=1}^{r} k_{i}\right), \quad \operatorname{deg} F\left(C_{1}^{\prime \prime}, t\right)=(q-2)\left(\left|E_{1}^{\prime \prime}\right|-\sum_{i=r+1}^{m} k_{i}\right) .
$$

Since $K\left[E_{1}^{\prime}\right]$ and $K\left[E_{1}^{\prime \prime}\right]$ are polynomial rings in disjoint sets of variables $E_{1}^{\prime}$ and $E_{1}^{\prime \prime}$, according to [25, Proposition 2.2.20, p. 42], we have an isomorphism

$$
C_{1}^{\prime} \otimes_{K} C_{1}^{\prime \prime} \simeq K\left[t_{1}, \ldots, t_{s-1}\right] /\left(t_{1}^{q-1}, \ldots, t_{s-1}^{q-1}, I_{1}(X), \ldots, I_{m}(X)\right)=S /\left(t_{s}, I(X)\right) .
$$

Altogether, as $F\left(C_{1}^{\prime} \otimes_{K} C_{1}^{\prime \prime}, t\right)=F\left(C_{1}^{\prime}, t\right) F\left(C_{1}^{\prime \prime}, t\right)$ (see [25, p. 102]), we obtain

$$
\begin{aligned}
\operatorname{reg} S / I(X) & =\operatorname{deg} F\left(S /\left(t_{s}, I(X)\right), t\right)=\operatorname{deg} F\left(C_{1}^{\prime} \otimes_{K} C_{1}^{\prime \prime}, t\right)=\operatorname{deg} F\left(C_{1}^{\prime}, t\right)+\operatorname{deg} F\left(C_{1}^{\prime \prime}, t\right) \\
& =(q-2)\left(\left|E_{1}^{\prime}\right|+\left|E_{1}^{\prime \prime}\right|-\sum_{i=1}^{m} k_{i}\right)=(q-2)\left(s-\sum_{i=1}^{m} k_{i}-1\right),
\end{aligned}
$$

as required. This completes the proof of case (II).

\section{REFERENCES}

[1] B. Bollobás, Modern Graph Theory, Graduate Texts in Mathematics 184 Springer-Verlag, New York, 1998.

[2] P. Delsarte, J. M. Goethals and F. J. MacWilliams, On generalized Reed-Muller codes and their relatives, Information and Control 161970 403-442.

[3] I. M. Duursma, C. Rentería and H. Tapia-Recillas, Reed-Muller codes on complete intersections, Appl. Algebra Engrg. Comm. Comput. 11 (2001), no. 6, 455-462.

[4] D. Eisenbud, The geometry of syzygies: A second course in commutative algebra and algebraic geometry, Graduate Texts in Mathematics 229, Springer-Verlag, New York, 2005.

[5] D. Eisenbud and B. Sturmfels, Binomial ideals, Duke Math. J. 84 (1996), 1-45.

[6] A. V. Geramita, M. Kreuzer and L. Robbiano, Cayley-Bacharach schemes and their canonical modules, Trans. Amer. Math. Soc. 339 (1993), no. 1, 163-189.

[7] L. Gold, J. Little and H. Schenck, Cayley-Bacharach and evaluation codes on complete intersections, J. Pure Appl. Algebra 196 (2005), no. 1, 91-99.

[8] M. González-Sarabia, J. Nava, C. Rentería and E. Sarmiento, Parameterized codes over cycles, preprint.

[9] M. González-Sarabia, C. Rentería and M. Hernández de la Torre, Minimum distance and second generalized Hamming weight of two particular linear codes, Congr. Numer. 161 (2003), 105-116.

[10] M. González-Sarabia and C. Rentería, Evaluation codes associated to complete bipartite graphs, Int. J. Algebra 2 (2008), no. 1-4, 163-170. 
[11] M. González-Sarabia, C. Rentería and H. Tapia-Recillas, Reed-Muller-type codes over the Segre variety, Finite Fields Appl. 8 (2002), no. 4, 511-518.

[12] D. Grayson and M. Stillman, Macaulay2, a software system for research in algebraic geometry. Available at http://www.math.uiuc.edu/Macaulay2/

[13] J. Harris, Algebraic Geometry. A first course, Graduate Texts in Mathematics 133, Springer-Verlag, New York, 1992.

[14] E. Miller and B. Sturmfels, Combinatorial Commutative Algebra, Graduate Texts in Mathematics 227, Springer, 2004.

[15] C. Rentería, A. Simis and R. H. Villarreal, Algebraic methods for parameterized codes and invariants of vanishing ideals over finite fields, Finite Fields Appl. 17 (2011), no. 1, 81-104.

[16] E. Reyes, R. H. Villarreal and L. Zárate, A note on affine toric varieties, Linear Algebra Appl. 318 (2000), 173-179.

[17] E. Sarmiento, M. Vaz Pinto and R. H. Villarreal, The minimum distance of parameterized codes on projective tori, Appl. Algebra Engrg. Comm. Comput. 22 (2011), no. 4, 249-264.

[18] E. Sarmiento, M. Vaz Pinto and R. H. Villarreal, On the vanishing ideal of an algebraic toric set and its parameterized linear codes, J. Algebra Appl., to appear. Preprint, 2011, arXiv:1107.4284v2 [math.AC].

[19] A. Sørensen, Projective Reed-Muller codes, IEEE Trans. Inform. Theory 37 (1991), no. 6, 1567-1576.

[20] R. Stanley, Hilbert functions of graded algebras, Adv. Math. 28 (1978), 57-83.

[21] H. Stichtenoth, Algebraic function fields and codes, Graduate Texts in Mathematics 254, Springer, 2010.

[22] S. Tohăneanu, Lower bounds on minimal distance of evaluation codes, Appl. Algebra Engrg. Comm. Comput. 20 (2009), no. 5-6, 351-360.

[23] M. Tsfasman, S. Vladut and D. Nogin, Algebraic geometric codes: basic notions, Mathematical Surveys and Monographs 139, American Mathematical Society, Providence, RI, 2007.

[24] M. Vaz Pinto and R. H. Villarreal, The degree and regularity of vanishing ideals of algebraic toric sets over finite fields. Preprint, 2011, arXiv:1110.2124 v1 [math.AC].

[25] R. H. Villarreal, Monomial Algebras, Monographs and Textbooks in Pure and Applied Mathematics 238, Marcel Dekker, New York, 2001.

CMuC, Department of Mathematics, University of Coimbra 3001-454 Coimbra, Portugal.

E-mail address: neves@mat.uc.pt

Departamento de Matemática, instituto Superior Técnico, Universidade Técnica de Lisboa, Avenida Rovisco Pais, 1, 1049-001 Lisboa, Portugal

E-mail address: vazpinto@math.ist.utl.pt

Departamento de Matemáticas, Centro de Investigación y de Estudios Avanzados del IPN, Apartado Postal 14-740, 07000 Mexico City, D.F.

E-mail address: vila@math.cinvestav.mx 